\title{
WASABI: a dynamic iterative framework for gene regulatory network inference
}

\author{
Arnaud Bonnaffoux ${ }^{1,2,3^{*}}$ (D) Ulysse Herbach ${ }^{1,2,4}$, Angélique Richard ${ }^{1}$, Anissa Guillemin ${ }^{1}$, \\ Sandrine Gonin-Giraud ${ }^{1}$, Pierre-Alexis Gros ${ }^{3}$ and Olivier Gandrillon ${ }^{1,2}$
}

\begin{abstract}
Background: Inference of gene regulatory networks from gene expression data has been a long-standing and notoriously difficult task in systems biology. Recently, single-cell transcriptomic data have been massively used for gene regulatory network inference, with both successes and limitations.

Results: In the present work we propose an iterative algorithm called WASABI, dedicated to inferring a causal dynamical network from time-stamped single-cell data, which tackles some of the limitations associated with current approaches. We first introduce the concept of waves, which posits that the information provided by an external stimulus will affect genes one-by-one through a cascade, like waves spreading through a network. This concept allows us to infer the network one gene at a time, after genes have been ordered regarding their time of regulation. We then demonstrate the ability of WASABI to correctly infer small networks, which have been simulated in silico using a mechanistic model consisting of coupled piecewise-deterministic Markov processes for the proper description of gene expression at the single-cell level. We finally apply WASABI on in vitro generated data on an avian model of erythroid differentiation. The structure of the resulting gene regulatory network sheds a new light on the molecular mechanisms controlling this process. In particular, we find no evidence for hub genes and a much more distributed network structure than expected. Interestingly, we find that a majority of genes are under the direct control of the differentiation-inducing stimulus.

Conclusions: Together, these results demonstrate WASABI versatility and ability to tackle some general gene regulatory networks inference issues. It is our hope that WASABI will prove useful in helping biologists to fully exploit the power of time-stamped single-cell data.
\end{abstract}

Keywords: Single-cell transcriptomics, Gene network inference, Multiscale modelling, Proteomic, High parallel computing, T2EC, Erythropoiesis

\section{Background}

It is widely accepted that the process of cell decision making results from the behavior of an underlying dynamic gene regulatory network (GRN) [1]. The GRN maintains a stable state but can also respond to external perturbations to rearrange the gene expression pattern in a new relevant stable state, such as during a differentiation process. Its identification has raised great expectations for practical applications in network medicine [2] like somatic cells

\footnotetext{
*Correspondence: a.bonnaffoux@vidium-solutions.com

'University Lyon, ENS de Lyon, University Claude Bernard, CNRS UMR 5239,

INSERM U1210, Laboratory of Biology and Modelling of the Cell, Lyon, France

${ }^{2}$ Inria Team Dracula, Inria Center Grenoble Rhône-Alpes, Lyon, France

Full list of author information is available at the end of the article
}

[3-5] or cancer cells reprogramming [6, 7]. The inference of such GRNs has, however, been a long-standing and notoriously difficult task in systems biology.

GRN inference was first based upon bulk data [8] using transcriptomics acquired through micro array or RNA sequencing (RNAseq) on populations of cells. Different strategies has been used for network inference including dynamic Bayesian networks $[9,10]$, boolean networks [11-13] and ordinary differential equations (ODE) [14] which can be coupled to Bayesian networks [15].

More recently, single-cell transcriptomic data, especially RNAseq [16], have been massively used for GRN inference (see $[17,18]$ for recent reviews). The arrival of those single-cell techniques led to question the fundamental limitations in the use of bulk data. Observations (c) The Author(s). 2019 Open Access This article is distributed under the terms of the Creative Commons Attribution 4.0 International License (http://creativecommons.org/licenses/by/4.0/), which permits unrestricted use, distribution, and reproduction in any medium, provided you give appropriate credit to the original author(s) and the source, provide a link to the Creative Commons license, and indicate if changes were made. The Creative Commons Public Domain Dedication waiver (http://creativecommons.org/publicdomain/zero/1.0/) applies to the data made available in this article, unless otherwise stated. 
at the single-cell level demonstrated that any and every cell population is very heterogeneous [19-21]. Two different interpretations of the reasons behind single-cell heterogeneity led to two different research directions:

1. In the first view, this heterogeneity is nothing but a noise that blurs a fundamentally deterministic smooth process. This noise can have different origins, like technical noise ("dropouts") or temporal desynchronization as during a differentiation process. This view led to the re-use of the previous strategies and was at the basis of the reconstruction of a "pseudo-time" trajectory (reviewed in [22]). For example, SingleCellNet [23] and BoolTraineR [24] are based on boolean networks with preprocessing for cell clustering or pseudo-time reconstruction. Such asynchronous Boolean network models have been successfully applied in [25]. Other probabilistic algorithms such as SCOUP [26], SCIMITAR [27] or AR1MA1-VBEM [28] also use pseudo-time reconstruction complemented with correlation analysis. ODE based methods can be exemplified with SCODE [29] and InferenceSnapshot [30] algorithms which also use pseudo-time reconstruction.

2. The other view is based upon a representation of cells as dynamical systems [31,32]. Within such a frame of mind, "noise" can be seen as the manifestation of the underlying molecular network itself. Therefore cellto-cell variability is supposed to contain very valuable information regarding the gene expression process [33]. This view was advocated among others by [34], suggesting that heterogeneity is rooted into gene expression stochasticity, and that cell state dynamic is a highly stochastic process due to bursting that jumps discontinuously between micro-states. Dynamic algorithms like SINCERITIES [35] are based upon comparison of gene expression distributions, incorporating (although not explicitly) the bursty nature of gene expression. We have recently described a more explicit network formulation view based upon the coupling of probabilistic two-state models of gene expression [36]. We devised a statistical hidden Markov model with interpretable parameters, which was shown to correctly infer small two-gene networks [36].

Despite their contributions and successes, all existing GRN inference approaches are confronted to some limitations:

1. The inference of interactions through the calculation of correlation between gene expression, whether based upon or linear [27] or non-linear [26] assumptions, is problematic. Such correlations can only reproduce events that have been previously observed. As a consequence, predictions of GRN response to new stimulus or modifications is not possible. Furthermore, correlation should not be mistaken for causality. The absence of causal relationship severely hampers any predictive ability of the inferred GRN.
2. The very possibility of making predictions relies upon our ability to simulate the behavior of candidate networks. This implicitly implies that network topologies are explicitly defined. Nevertheless, several inference algorithms [27-29, 35] propose a set of possible interactions with independent confidence levels, generally represented by an interaction matrix. The number of possible actionable networks deduced from combining such interactions is often too large to be simulated.

3. Regulatory proteins within a GRN are usually restricted to transcription factors (TF), like in [24, 26-30]. Possible indirect interactions are completely ignored. A trivial example is a gene encoding a protein that induces the nuclear translocation of a constitutive TF. In this case, the regulator gene will indirectly regulate TF target genes, and its effect will be crucial in understanding the GRN behavior.

4. Most single-cell inference algorithms rely upon the use of a single type of data, namely transcriptomics. By doing so, they implicitly assume protein levels to be positively correlated with RNA amounts, which has been proven to be wrong in case of post-translational regulation (see [33] for an illustration in circadian clock). Besides, at single-cell scale, mRNA and proteins typically have a poor linear correlation [34], even in the absence of post-translational regulation.

5. The choices of biological assumptions are also important for the biological relevance of GRN models. The use of statistical tools can be really powerful to handle largescale network inference problem with thousand of genes, but the price to pay is loss of biological representativeness. By definition a model is a simplification of the system, but when simplifying assumptions are induced by mathematical tools, like linear [27-29, 35] or binary (boolean) requirements $[23,24]$, the model becomes solvable at the expense of its biological relevance.

In the present work we address the above limitations and we propose an iterative algorithm called WASABI, dedicated to inferring a causal dynamical network from time-stamped single-cell transcriptomic data, with the capability to integrate protein measurements. In the first part we present the WASABI framework which is based upon a mechanistic model for gene-gene interactions [36]. In the second part we benchmark our algorithm using in silico GRNs with realistic gene parameter values. Finally we apply WASABI on our in vitro data [37] and analyze the resulting GRN candidates.

\section{Results}

Our goal is to infer causalities involved in GRN through analysis of dynamic multi-scale/level data with the help of a mechanistic model [36]. We first present an overview of the WASABI principles and framework. We then benchmark its ability to correctly infer in silico-generated toy 
GRNs. Finally, we apply WASABI on our in vitro data on avian erythroid differentiation model [38] to generate biologically relevant GRN candidates.

\section{WASABI inference principles and implementation}

WASABI stands for "WAveS Analysis Based Inference". It is a framework built on a novel inference strategy based on the concept of "waves". We posit that the information provided by an external stimulus will affect genes one- by-one through a cascade, like waves spreading through a network (Fig. 1-a). This wave process harbors an inertia determined by mRNA and protein half-lives which are given by their degradation rate.

By definition, causality is the link between cause and consequence, and causes always precede consequences. This temporal property is therefore of paramount importance for causality inference using dynamic data. In our mechanistic and stochastic model of GRN [36] (detailed

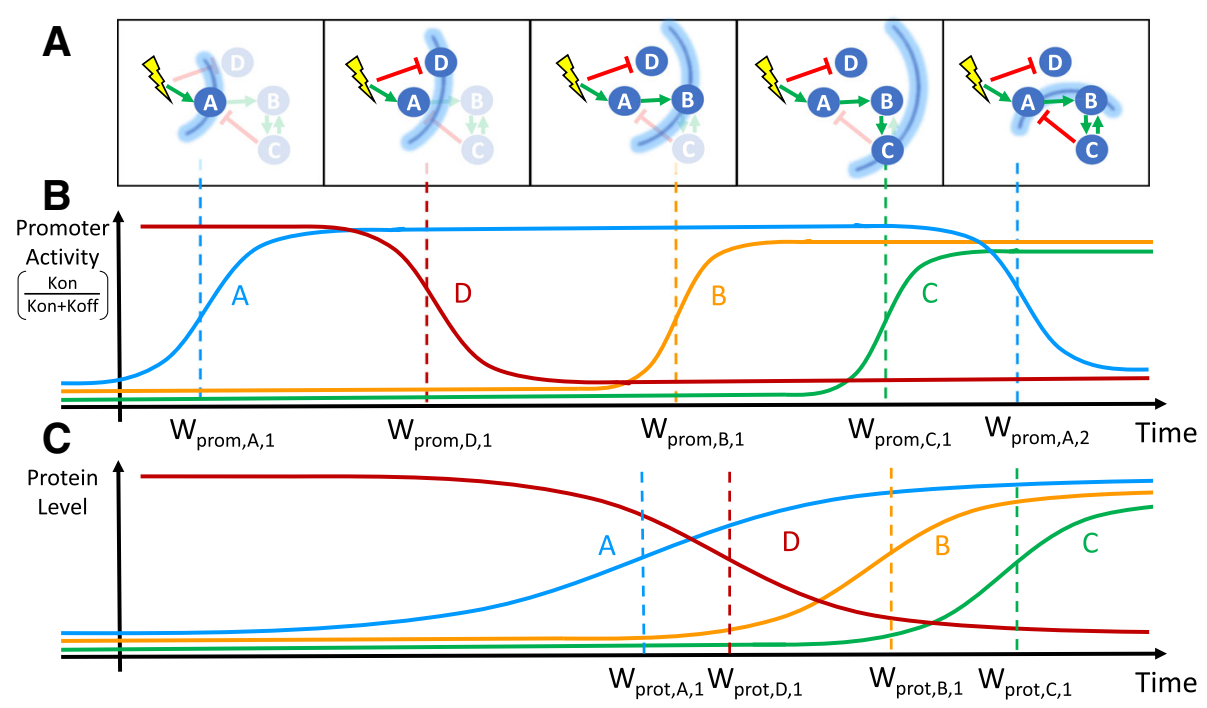

D

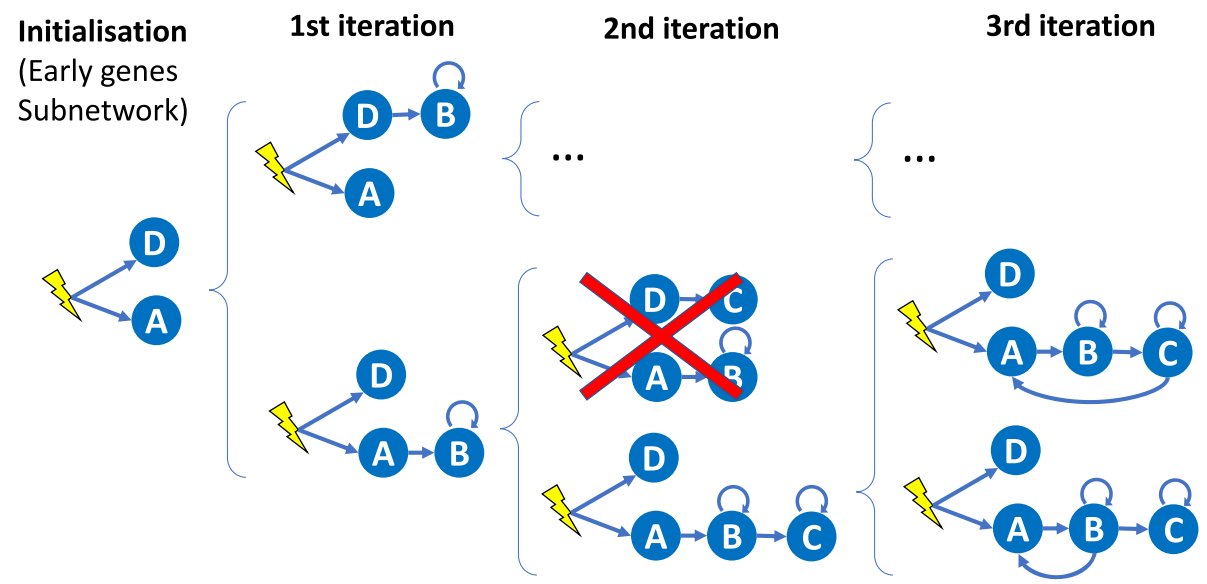

Fig. 1 WASABI at a glance. a Schematic view of a GRN: the stimulus is represented by a yellow flash, genes by blue circles and interactions by green (activation) or red (inhibition) arrows. The stimulus-induced information propagation is represented by blue arcs corresponding to wave times. Genes and interactions that are not affected by information at a given wave time are shaded. At wave time 5 , gene $C$ returns information on gene $A$ and $B$ by feedback interaction creating a backflow wave. b Promoter wave times: Promoter wave times correspondto inflections point of gene promoter activity defined as the $k_{\mathrm{on}} /\left(k_{\mathrm{on}}+k_{\mathrm{off}}\right)$ ratio. c Protein wave times: Protein wave times correspondto inflections point of mean protein level. d Inference process. Blue arrows represent interactions selected for calibration. Based on promoter waves classification genes are iteratively added to sub-GRN previously inferred to get new expanded GRN. Calibration is performed by comparison of marginal RNA distributions between in silico and in vitro data. Inference is initialized with calibration of early genes interaction with stimulus, which gives initial sub-GRN. Latter genes are added one by one to a subset of potential regulators for which a protein wave time is close enough to the added gene promoter wave time. Each resulting sub-GRN is selected regarding its fit distance to in vitro data. If fit distance is too important sub-GRN can be eliminated (red cross). An important benefit of this process is the possibility to parallelize the sub-GRN calibrations over several cores, which results in a linear computational time regarding the number of genes. Note that only a fraction of all tested sub-GRN is shown 
in "Methods" section Fig. 7), the cause corresponds either to the protein of the regulating gene or a stimulus, which level modulates as a consequence the promoter state switching rates $k_{\text {on }}$ (i.e. probability to switch from inactive to active state) and $k_{\text {off }}$ (active to inactive) of the target gene. A direct consequence of causality principle for GRNs is that a dynamical change in promoter activity can only be due to a previous perturbation of a regulating protein or stimulus. For example, assuming that the system starts at a steady-state, early activated genes (referred to as early genes) can only be regulated by the stimulus, because it is the only possible cause for their initial evolution. An illustration is given in Fig. 1-a: gene $A$ initial variation can only be due to the stimulus and not by the feedback from gene $C$, which will occur later. A generalization of these concepts is that for a given time after the stimulus, we can infer the subnetwork composed exclusively by genes affected by the spreading of information up to this time. Therefore we can infer iteratively the network by adding one gene at a time (Fig. 1-d) regarding their promoter wave time order (Fig. 1-b) and comparing with protein wave time of previous added genes (Fig. 1-c).

For this, we need to estimate promoter and protein wave times for each gene and then sort them by promoter wave time. We define the promoter activity level by the $k_{\text {on }} /\left(k_{\text {on }}+k_{\text {off }}\right)$ ratio, which corresponds to the local mean active duration (Fig. 1-b). Promoter wave time is defined as the inflection time point of promoter activity level where $50 \%$ of evolution between minimum and maximum is reached. Since promoter activity is not observable, we estimate the inflection time point of mean RNA level from single-cell transcriptomic kinetic data [37], and retrieve the delay induced by RNA degradation to deduce promoter wave time. Protein wave times correspond to the inflection point of mean protein level, which can be directly observed with our proteomic data [39]. A detailed description of promoter and protein wave time estimation can be found in the "Methods" section. One should note that a gene can have more than one wave time in case of non monotonous variation of promoter activity, due to feedbacks (like gene $A$ in our example) or incoherent feed-forward loop.

The WASABI inference process (Fig. 1-c) takes advantage of the gene wave time sorting by adopting a divide and conquer strategy. We remind that a main assumption of our interaction model is the separation between mRNA and protein timescales [36]. As a consequence, for a given interaction between a regulator gene and a regulated gene, the regulated promoter wave time should be compatible with the regulator protein wave time. At each step, WASABI proposes a list of possible regulators in order to reduce the dimension of the inference problem. This list is limited to regulators with compatible protein wave time within the range of 30 hours before and 20 hours after the promoter wave time of the added regulated gene. This constraint has been set up from in silico study (see next section). For example, in Fig. 1, gene $B$ can be regulated by gene $A$ or $D$ since their protein wave time are close to gene $B$ promoter wave time. Gene $C$ can be regulated by gene $B$ or $D$, but not $A$ because its protein wave time is too earlier compared to gene $C$ promoter wave time.

For new proposed interactions, a typical calibration algorithm can be used to finely tune interaction parameter in order to fit simulated mRNA marginal distribution with experimental marginal distribution from transcriptomic single-cell data. To avoid over-fitting issues, only efficiency interaction parameter $\theta_{i, j}$ (Fig. 7) is tuned. To estimate fitting quality we define a GRN fit distance based on the Kantorovitch distances between simulated and experimental mRNA marginal distributions (please refer to "Methods" section for a detailed description of interaction function and calibration process). If the resulting fitting is judged unsatisfactory (i.e. GRN fit distance is greater than a threshold), the sub-GRN candidate is pruned. For genes presenting several waves, like gene $A$, each wave will be separately inferred. For example, gene $A$ initial increase is fitted during initialization step, but only the first experimental time points during promoter activity increase will be used for calibration. Genes $B$ and $C$ regulated after gene $A$ up-regulation will be added to expand sub-GRN candidates. Finally, the wave corresponding to gene $A$ down-regulation is then fitted considering possible interactions with previously added genes (namely gene $B$ and $C$ ), which permits the creation of feedback loops or incoherent feed-forward loops.

Positive feedback loops cannot be easily detected by wave analysis because they only accelerate, and eventually amplify, gene expression. Yet, their inference is important for the GRN behavior since they create a dynamic memory and, for example, may thus participate to irreversibility of the differentiation process. To this end, we developed an algorithm to detect the effect of positive feedback loops on gene distribution before the iterative inference (see Supporting information). We modeled the effect of positive feedback loops by adding auto-positive interactions. Note that such a loop does not necessarily mean that the protein directly activates its own promoter: it simply means that the gene is influenced by a positive feedback, which can be of different nature. For example, in the GRN presented in Fig. 1-a, genes $B$ and $C$ mutually create a positive feedback loop. If this positive feedback loop is detected we consider that each gene has its own auto-positive interaction as illustrated in Fig. 1-c. Positive feedback loops could also arise from the existence of self-reinforcing open chromatin states [40] or be due to the fact that binding of one TF can shape the DNA in a manner that it promotes the binding of the second TF [41]. 


\section{In silico benchmarking}

We decided to first calibrate and then assess WASABI performance in a controlled and representative setting.

\section{Calibration of inference parameters}

In the first phase we assessed some critical values to be used in the inference process. We generate realistic GRNs (Fig. 2-a) where 20 genes from in vitro data were randomly selected with associated in vitro estimated parameters (see Supporting information). Interactions were randomly defined in order to create cascade networks with no feedback nor auto-positive feedback as an initial assessment phase.

We limited ourselves to 4 network levels (with 5 genes at each level, see Fig. 2-a for an example) because we observed that the information provided by the stimulus is almost completely lost after 4 successive interactions in the absence of positive feedback loops. This is very likely caused by the fact that each gene level adds both some intrinsic noise, due to the bursty nature of gene expression, as well as a filtering attenuation effect due to RNA and protein degradation.

We first analyzed the special case of early genes that are directly regulated by the stimulus (Fig. 2-b). Their promoter wave times were lower than all other genes but one. Therefore we can identify early genes with good confidence, based on comparison of their promoter wave time with a threshold. Given these in silico results, we then decided in the WASABI pre-processing step to assume that genes with a promoter wave time below $5 \mathrm{~h}$ must be early genes, and that genes with a promoter wave time larger than $7 \mathrm{~h}$ can not be early genes. Interactions between the stimulus and intermediate genes, with promoter wave times between $5 \mathrm{~h}$ and $7 \mathrm{~h}$, have to be tested during the inference iterative process and preserved or not.
We then assessed what would be the acceptable bounds for the difference between regulator protein wave time and regulated gene promoter activity. Ten in silico cascade GRNs were generated and simulated for 500 cells to generate population data from which both protein and promoter wave times were estimated for each gene. Based on these data, we computed the difference between estimated regulated promoter wave time minus its regulator protein wave time for all interactions in all networks. The distribution of these wave differences is given in Fig. 2-c. One can notice that some wave differences had negative values. This is due to the shape of the Hill interaction function (see Eq. 3 in "Methods" section) with a moderate transition slope $(\gamma=2)$. If the protein threshold (which corresponds to typical EC50 value) is too close to the initial protein level, then a slight protein increase will activate target promoter activity. Therefore, promoter activity will be saturated before regulator protein level and thus the difference of associated wave times is negative. This shows that one can accelerate or delay information, depending on the protein threshold value. In order to be conservative during the inference process, we set the RNA/Protein wave difference bounds to $[-20 \mathrm{~h} ; 30 \mathrm{~h}]$ in accordance with the distribution in Fig. 2-c. One should note that this range, even if conservative, already removes two thirds of all possible interactions, thereby reducing the inference complexity.

We finally observed that for interactions with genes harboring an auto-positive feedback, wave time differences could be larger. In this case, wave difference bounds were estimated to [-30h, 50h] (see supporting information). We interpret this enlargement by an under-sampling time resolution problem since auto-positive feedback results in a sharper transition. As a consequence, promoter state transition from inactive to active is much faster: if it happens between two experimental time points, we cannot detect precisely its wave time.

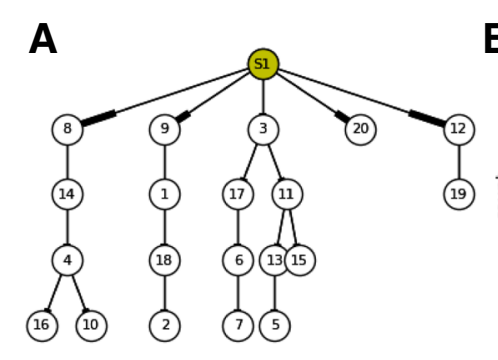

2 Cascade in silico GRN a Cascade GRN types are generated to study wave early genes (blue) with other genes (red). Displayed are promoter waves with a wave time lower than $15 \mathrm{~h}$ for graph clarity. c For each interactions of 10 in silico GRNs we compute the difference between estimated regulated promoter wave time minus its regulator protein wave time. Distribution of promoter/protein wave time difference is given for all interactions of all in silico GRNs 


\section{Inference of in silico GRNs}

WASABI was then tested for its ability to infer in silico GRNs (complete definition in supporting information) from which we previously simulated experimental data for mRNA and protein levels at single-cell and population scales. We first assessed the simplest scenario with a toy GRN composed of two branches with no feedback (a cascade GRN; Fig. 3-a). The GRN was limited to 6 genes and to 3 levels in order to reduce computational constraints. Nevertheless, even in such a simple case, the inference problem is already a highly complex challenge with more than $10^{20}$ possible directed networks.

Wave times were estimated for each gene from simulated population data for RNA and protein (data available in supporting information). Table 1 provides estimated waves time for the cascade GRN. It is clear that the gene network level is correctly reproduced by wave times.

We then ran WASABI on the generated data and obtained 88 GRN candidates (Fig. 3-b). The huge reduction in numbers (from $10^{20}$ to 88 ) illustrates the power of WASABI to reduce complexity by applying our wavesbased constraints. We defined two measures for further assessing the relevance of our candidates:

1. Quality quantifies proportion of real interactions that are conserved in the candidate network (see supporting information for a detailed description). A $100 \%$ corresponds to the true GRN.

2. A fit distance, defined as the mean of the 3 worst gene fit distances, where gene fit distance is the mean of the 3 worst Kantorovitch distances [42] among time points (see the "Methods" section).

We observed a clear trend that higher quality is associated with a lower fit distance (Fig. 3-b), which we denote as a good specificity. When inferring in vitro GRNs, one does not have access to quality score, contrary to fit distance. Hence, having a good specificity enables to confidently estimate the quality of GRN candidates from their fit distance. Thus, this result demonstrates that our fit distance criterion can be used for GRN inference. Nevertheless, even in the case of a purely in silico approach, quality and fit distance can not be linked by a linear relationship. In other words, the best fit distance can not be taken for the

Table 1 Wave times

\begin{tabular}{llll}
\hline GRN & Gene & Wromoter & $W_{\text {protein }}$ \\
\hline & 4 & 4.12 & 12.99 \\
& 1 & 4.26 & 22.33 \\
Cascade & 5 & 15.19 & 45.50 \\
& 2 & 17.67 & 44.88 \\
& 3 & 37.88 & 60.10 \\
& 6 & 40.06 & 60.72 \\
\hline
\end{tabular}

Promoter and protein wave times (in hours) estimated from in silico simulated data best quality (see below for other toy GRNs). This is likely to be due to both the stochastic gene expression process as well as the estimation procedure. We therefore needed to estimate an acceptable maximum fit distance threshold for true GRN. For this, we ran directed inferences, where WASABI was informed beforehand of the true interactions, but calibration was still run to calibrate interaction parameters. We ran 100 directed inferences and defined the maximum acceptable fit distance (Fig. 3-c) as the distance for which $95 \%$ of true GRN fit distance was below. This threshold could also be used as a pruning threshold (green dashed line in Fig. 3-b) in subsequent iterative inferences, thereby progressively reducing the number of acceptable candidates. We then analyzed a situation where we added either an auto-activation loop or a negative feedback (Fig. 4-a and c and supporting information for estimated wave times).

In both cases, GRN inference specificity was lower than for cascade network inference. Nevertheless in both cases the true network was inferred and ranked among the first candidates regarding their fit distance (Fig. 4-b and d), demonstrating that WASABI is able to infer auto-positive and negative feedback patterns. However there were more candidates below the acceptable maximum fit distance threshold and there was no obvious correlation between high quality and low fit distance. We think it could be due to data under-sampling regarding the network dynamics (see upper and discussion).

\section{In vitro application of WASABI}

We then applied WASABI on our in vitro data, which consists in time stamped single-cell transcriptomic [37] and bulk proteomic data [39] acquired during T2EC differentiation [38], to propose relevant GRN candidates.

We first estimated the wave times (Fig. 5). Promoter waves ranged from very early genes regulated before $1 \mathrm{~h}$ to late genes regulated after $60 \mathrm{~h}$. Promoter activity appeared bimodal with an important group of genes regulated before $20 \mathrm{~h}$ and a second group after $30 \mathrm{~h}$. Protein wave distribution was more uniform from $10 \mathrm{~h}$ to $60 \mathrm{~h}$, in accordance with a slower dynamics for proteins. Remarkably, 10 genes harbored non-monotonous evolution of their promoter activity with a transient increase. It can be explained by the presence of a negative feedback loop or an incoherent feed-forward interaction. These results demonstrate that real in vitro GRN exhibits distinguishable "waves".

In order to limit computation time, we decided to further restrict the inference to the most important genes in term of the dynamical behavior of the GRN. We first detected 25 genes that are defined as early with a promoter time lower than $5 \mathrm{~h}$. We then defined a second class of genes called "readout" which are influenced by the network state but can not influence in return other genes. 


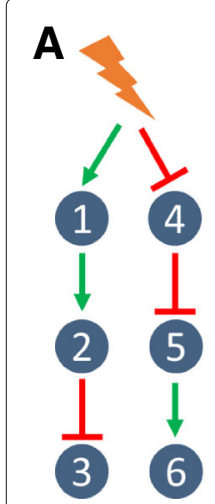

B

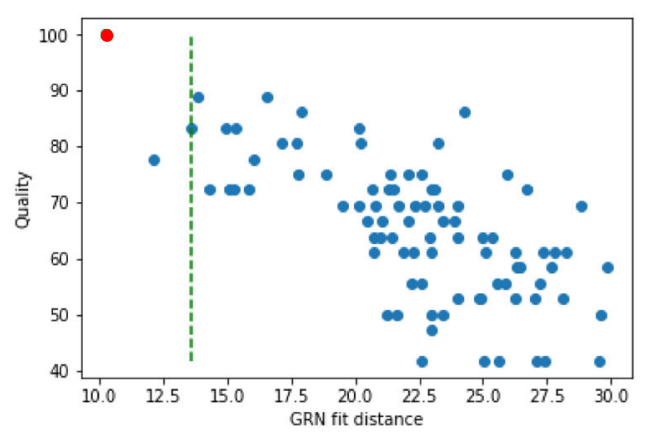

C

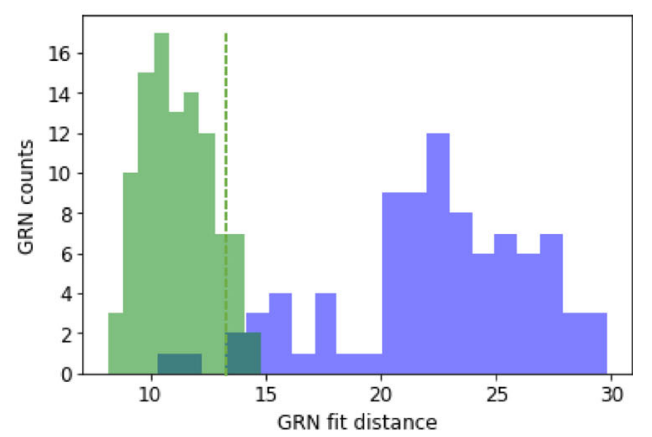

Fig. 3 In silico cascade GRN inference a The cascade GRN. Genes parameters were taken from in vitro estimations to mimic realistic behavior. Experimental data were generated to obtain time courses of transciptomic data, at single-cell and population scale, and also proteomic data at population scale. $\mathbf{b}$ WASABI was run to infer in silico cascade GRN and generated 88 candidates. A dot represents a network candidate with its associated fit distance and inference quality (percentage of true interactions). True GRN is inferred (red dot, 100\% quality). Acceptable maximum fit distance (green dashed line) corresponds to variability of true GRN fit distance. Its computation is detailed in figure C. Three GRN candidates (including the true one) have a fit distance below threshold. c Variability of true GRN fit distance (green dashed line in figures B and C) is estimated as the threshold where $95 \%$ of true GRN fit distance is below. Fit distance distribution is represented for true GRN (green) and candidates (blue) for cascade in silico GRN benchmark. True GRNs are calibrated by WASABI directed inference while candidates are inferred from non-directed inference. Fit distance represents similitude between candidates generated data and reference experimental data

A
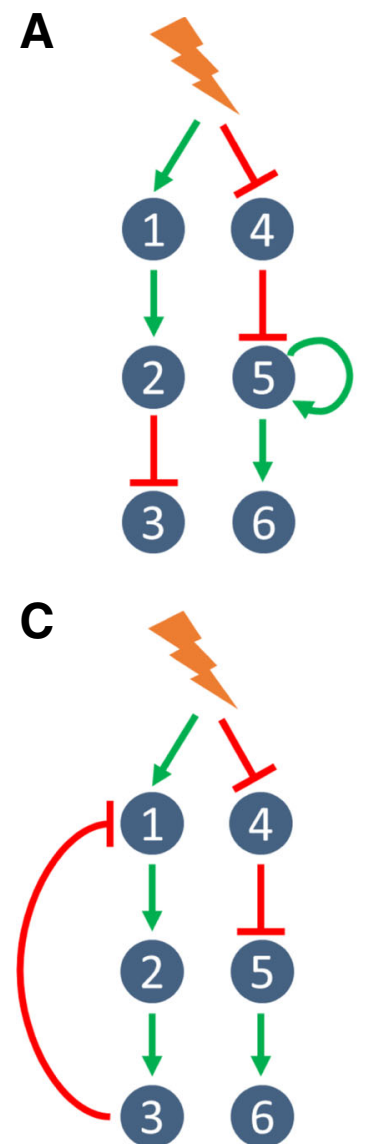

B

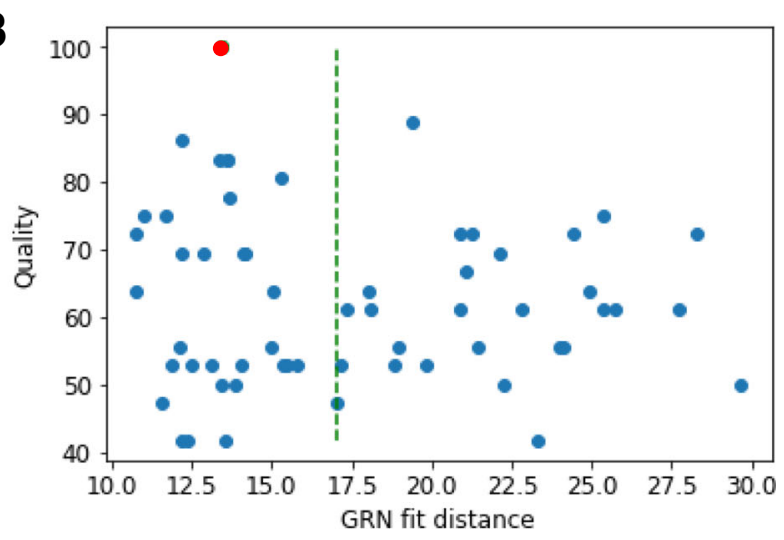

D

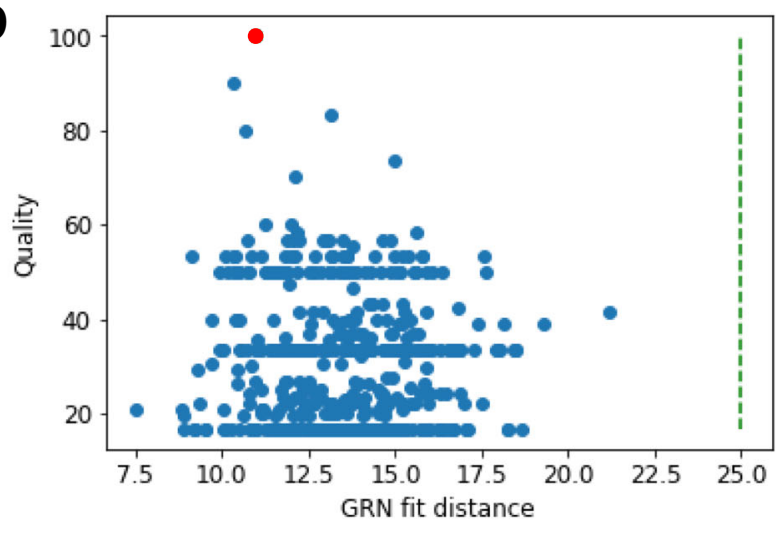

Fig. 4 In silico GRN with feedbacks a Addition of one positive feedback onto the cascade GRN. b WASABI was run to infer in silico cascade GRN with a positive feedback and generated 59 candidates, 31 of which having an acceptable fit distance. See legend to Fig. 3-b for details. c Addition of one negative feedback onto the cascade GRN. d WASABI was run to infer in silico cascade GRN with a negative feedback and generated 476 candidates, all of which having an acceptable fit distance. See legend to Fig. 3-b for details 

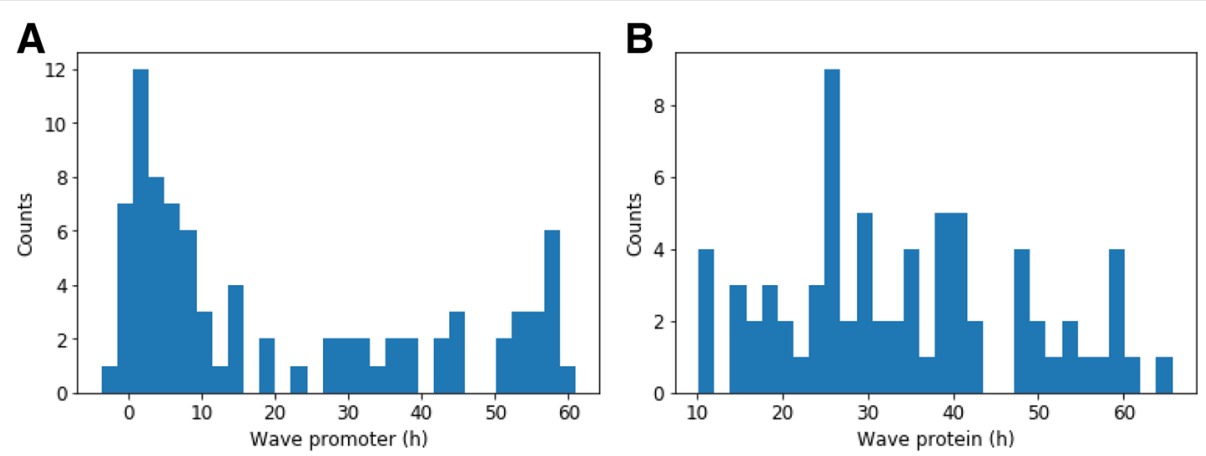

Fig. 5 Promoter and protein wave time distributions. Distribution of in vitro promoter (a) and protein (b) wave times for all genes estimated from RNA and proteomic data at population scale. Counts represent number of genes. Note: a gene can have several waves for its promoter or protein

Their role for final cell state is certainly crucial, but their influence on the GRN behavior is nevertheless limited. 41 genes were classified as readout so that 24 genes were kept for iterative inference, in addition to the 25 early genes. 9 of these 24 genes have 2 waves due to transient increase, which means that we have 33 waves to iteratively infer.

\section{In vitro GRN candidates}

After running for 16 days using 400 computational cores, WASABI returned a list of 381 GRN candidates. Candidate fit distances showed a very homogeneous distribution (see supporting information) with a mean value around 30, together with outliers at much higher distances. Removing those outliers left us with 364 candidates. Compared to inference of in silico GRN, in vitro fitting is less precise, as we could expect. But it is an appreciable performance and it demonstrates that our GRN model is relevant.

We then analyzed the extent of similarities among the GRN candidates regarding their topology by building a consensus interaction matrix (Fig. 6-a). The first observation is that the matrix is very sparse (except for early genes in first raw and auto-positive feedbacks in diagonal) meaning that a sparse network is sufficient for reproducing our in vitro data. We also clearly see that all candidate GRNs share closely related topologies. This is clearly obvious for early genes and auto-positive feedbacks. Columns with interaction rates lower than $100 \%$ correspond to latest integrated genes in the iterative inference process with gene index (from earlier to later) 70, 73, 89, 69 and 29. Results from existing algorithms are usually presented in such a form, where the percent of interactions are plotted [27-29, 35]. But one main advantage of our approach is that it actually proposes real GRN candidates, which may be individually examined.

We therefore took a closer look at the "best" candidate network, with the lowest Fit distance to the data (Fig. 6-b). We observed very interesting and somewhat unexpected patterns:
1. Most of the genes (84\%) with an auto-activation loop. As mentioned earlier, this was a consensual finding among the candidate networks. It is striking because typical GRN graphs found in the literature do not have such predominance of auto-positive feedbacks.

2. A very large number of genes were found to be early genes that are under the direct control of the stimulus. It is noticeable that most of them were found to be inhibited by the stimulus, and to control not more than one other gene at one next level.

3 . We previously described the genes whose product participates in the sterol synthesis pathway, as being enriched for early genes [37]. This was confirmed by our network analysis, with only one sterol-related gene not being an early gene.

4. Among 7 early genes that are positively controlled by the stimulus, 6 are influenced by an incoherent feedforward loop, certainly to reproduce their transient increase experimentally observed [37].

5. One important general rule is that the network depth is limited to 3 genes. One should note that this is not imposed by WASABI which can create networks with unlimited depth. It is consistent with our analysis on signal propagation properties in in silico GRN. If network depth is too large, signal is too damped and delayed to accurately reproduce experimental data.

6. One do not see network hubs in the classical sense. The genes in the GRNs are connected to at most four neighbors. The most impacting "node" is the stimulus itself.

7. One can also observe that the more one progress within the network, the less consensual the interaction are. Adding the leaves in the inference process might help to stabilize those late interactions.

Altogether those results show the power of WASABI to offer a brand-new vision of the dynamical control of differentiation.

\section{Discussion}

In the present work we introduced WASABI as a new iterative approach for GRN inference based on single- 


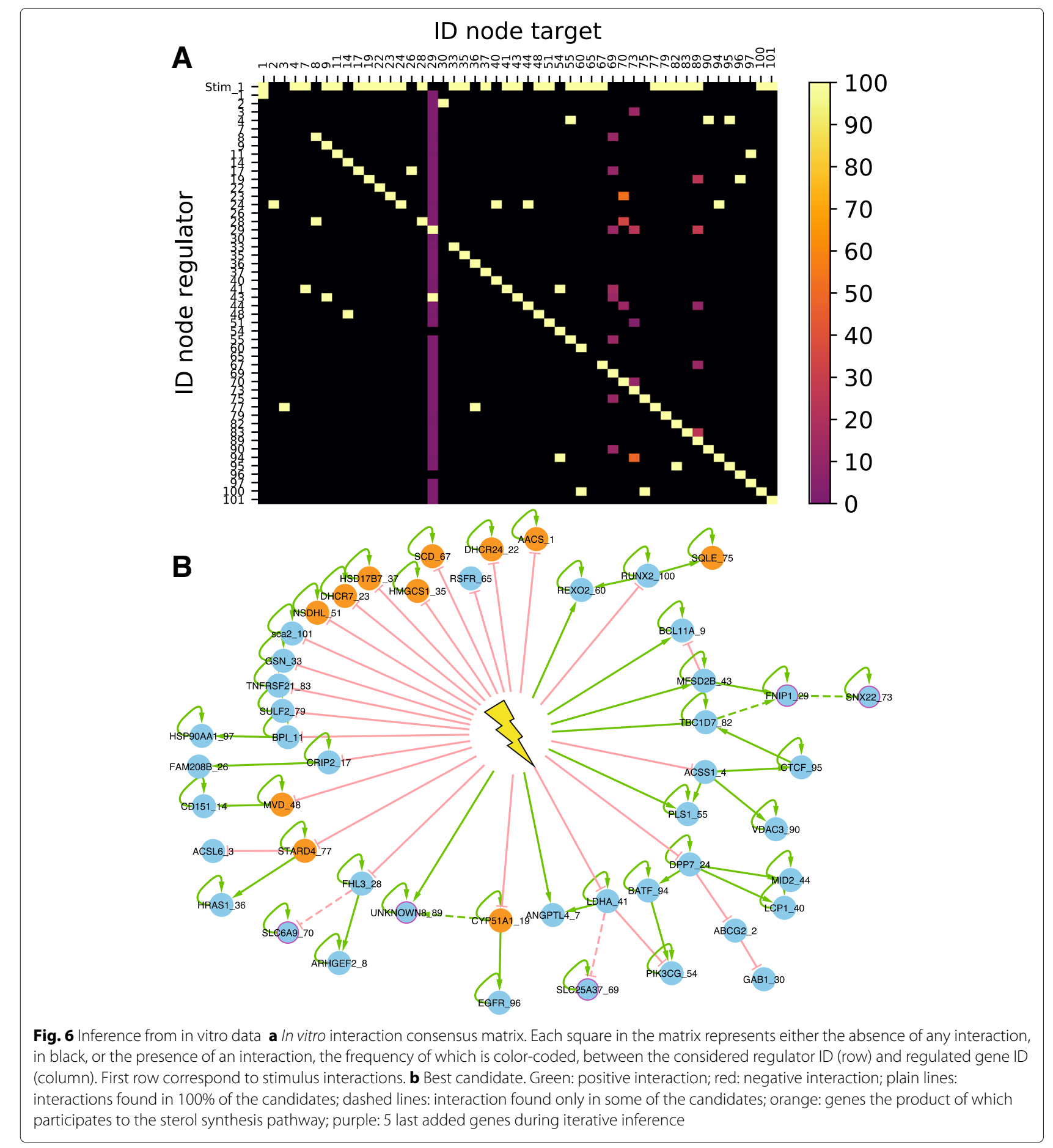

cell data. We benchmarked it on a representative in silico environment before its application on in vitro data.

\section{WASABI tackles GRN inference limitations}

Usually, to demonstrate that a new inference method outperforms previous ones benchmarking is performed [43-45]. However, evaluation of GRN inference methods is a problem per se due to the lack of a gold standard against which different algorithms might be benchmarked [46]. For example, typical in silico model like [47] are based on population deterministic behavior (only a Gaussian white-noise is added) and do not consider posttranslational regulation (degradation rates are constant). If we benchmark WASABI with other inference algorithm 
based on our GRN mechanistic model it is quite obvious that we will outperform other methods, for example just because we consider post-translational regulation integrating both transcriptomic and proteomic data, unlike other methods. Another point comes from the metric usually used to compare inference methods like ROC (Receiver Operating Characteristic). This metric focuses on the number of true inferred interactions instead of the overall network topology, or the dynamical network behavior.

More over, in our view it would be meaningless to compare our approach to any other approach that would not yield a representative executable model $[48,49]$ which most approach do not provide. For example, SINCERITIES [35] analyses single cell transcriptomic time-course data to reconstruct an interaction matrix, but this matrix is not executable and can not reproduce time series of transcriptomic data. Other methods, like Single Cell Network Synthesis toolkit [49] based on a boolean model, propose to reconstruct executable models from single cell data. However, to our knowledge, none of these executable methods is able to reproduce time series of experimental distribution observed at single cell level, which limits fundamentally they ability to produce testable predictions. We definitively consider that the only way to evaluate an inference algorithm is to experimentally validate its predictions. This is the reason why we are willing to couple WASABI with an iterative process of Design Of Experiment (DOE) as discussed later.

However, despite experimental validation, we are convinced that WASABI has the ability to tackle some general GRN inference issues based on the assumptions on which WASABI as been designed and on in silico validation results.

1. WASABI goes beyond mere correlations to infer causalities from time stamped data analysis as demonstrated on in silico benchmark (Fig. 3) even in the presence of circular causations (Fig. 4), based upon the principle that the cause precedes the effect.

2. Contrary to most GRN inference algorithms [27-29, 35] based upon the inference of interactions, WASABI is network centered and generates several candidates with explicitly defined networks topology (Fig. 6-b), which is required for prediction making and simulation capability. Generating a list of interactions and their frequency from such candidates is a trivial task (Fig. 6-a) whereas the reverse is usually not possible. Moreover, WASABI explicitly integrates the presence of an external stimulus, which surprisingly is never modeled in other approaches based on single-cell data analysis. It could be very instrumental for simulating for example pulses of stimuli.

3. WASABI is not restricted to TFs. Most of the in vitro genes we modeled are not TFs. This is possible thanks to the use of our mechanistic model [36] which integrates the notion of timescale separation. It assumes that every biochemical reaction such as metabolic changes, nuclear translocations or post-translational modifications are faster than gene expression dynamics (imposed by mRNA and protein half-life) and that they can be abstracted in the interaction between 2 genes. Our interaction model is therefore an approximation of the underlying biochemical cascade reactions. This should be kept in mind when interpreting an interaction in our GRN: many intermediaries (fast) reactions may be hidden behind this interaction.

4. Optionally, WASABI offers the capability to integrate proteomic data to reproduce translational or post-translational regulation. Our proteomic data [39] demonstrate that nearly half of detected genes exhibit mRNA/protein uncoupling during differentiation and allowed to estimate the time evolution of protein production and degradation rates. Nevertheless, we are not fully explanatory since we do not infer causalities of these parameters evolution. This is a source of improvement discussed later.

5. We deliberately developed WASABI in a "brute force" computational way to guarantee its biological relevance and versatility. This allowed to minimize simplifying assumptions potentially necessary for mathematical formulations. During calibration, we used a simple Euler solver to simulate our networks within model (1). This facilitates addition of any new biological assumption, like post-translation regulations, without modifying the WASABI framework, making it very versatile. Thanks to the splitting and parallelization allowed by WASABI original gene-by-gene iterative inference process, the inference problem becomes linear regarding the network size, whereas typical GRN inference algorithms face combinatorial curse. This strategy also allowed the use of High Parallel Computing (HPC) which is a powerful tool that remains underused for GRN inference [23, 50].

\section{WASABI performances, improvements and next steps}

WASABI has been developed and tested on an in silico controlled environment before its application on in vitro data. Each in silico network true topology was successfully inferred. Cascade type GRN is totally inferred (Fig. 3) with a good specificity. Auto-positive and negative feedback networks (Fig. 4) were also inferred, demonstrating WASABI's ability to infer circular causations, but specificity is lower. This might be due to a time sampling of experimental data being longer than the network dynamic time scale. Auto-positive feedback creates a switch like response, the dynamic of which is much quicker than simple activation. Thus, to capture accurately auto-positive feedback wave time, we should use high frequency time sample for RNA experimental data during auto-positive feedback activation short period. For 
negative feedback interactions, WASABI calibrated initial increase considering only first experimental time points before feedback effect. Consequently, precision of first interaction was decreased and more false positive subGRN candidates were selected. Increasing the frequency of experimental time sampling during initial phase should overcome this problem.

As it stands our mechanistic model is only accounting for transcriptional regulation through proteins. It does not take into account other putative regulation level, including translational or post-translational regulations, or regulation of the mRNA half-life, although there is ample evidence that such regulation might be relevant [51, 52]. Provided that sufficient data is available, it would be straightforward to integrate such information within the WASABI framework. For example, the estimation of the degradation rates at the single-cell level for mRNAs and proteins has recently been described [53], the distribution of which could then be used as an input into the WASABI inference scheme.

Cooperativity and redundancies are not considered in the current WASABI framework, so that a gene can only be regulated by one gene, except for negative feedback or incoherent feedforward interactions. However, many experimentally curated GRN show evidence for cooperations ( 2 genes are needed to activate a third gene) or redundant interactions ( 2 genes independently activating a third gene) [54]. We intentionally did not considered such multi-interactions because our current calibration algorithm relies on the comparison of marginal distributions which are not sufficiently informative for inferring cooperative effects. It is our belief that the use of joint distribution of two genes or more should enable such inference. We previously developed in our group a GRN inference algorithm which is based on joint distribution analysis [36] but which does not consider time evolution. We are therefore planning to integrate joint-distributionbased analyses within the WASABI framework in order to improve calibration, by upgrading the objective function with measurement considering joint-distribution comparison.

HPC capacities used during iterative inference impacts WASABI accuracy. Indeed late iterations are supposed more discriminative than the first one because false GRN candidates have accumulated too many wrong interactions so that calibration is not able to compensate for errors. However, if the expansion phase is limited by available computational nodes, the true candidate may be eliminated because at this stage inference is not discriminative enough. Therefore improving computing performances would represent an important refinement and we have initiated preliminary studies in that direction [50].

As it stands, WASABI is limited to infer networks with less than 100 genes in a reasonable time. However, by means of all improvements described above, WASABI can be upscaled to infer network with more than 1000 genes using recent sc-RNA-seq technologies [55]. This is achievable because WASABI inference computational time is linear regarding the number of genes. As a consequence, increasing the number of genes by one order of magnitude only imposes to decrease computational time by the same ratio, which is fairly workable.

Nevertheless, despite all possible improvements, GRN inference will remain per se an asymptotically solvable problem due to inferability limitations [56], intrinsic biological stochasticity, experimental noise and sampling. This is why we propose a set of GRN candidates with acceptable confidence level. A natural companion of the WASABI approach would be a phase of design of experiments (DOE) specifically aiming at selecting the most informative experiments to discriminate among the candidates. Such DOE procedures have already been developed for GRN inference, but none of them takes into account the mechanistic aspects and the stochasticity of gene expression [56, 57]. Extending the DOE framework to stochastic models is currently being developed in our group.

\section{New insights on typical GRN topology}

The application of WASABI on our in vitro model of differentiation generated several GRN candidates with a very interesting consensus topology (Fig. 6).

1. We can see that the stimulus (i.e. medium change [37]) is a central regulator of our GRN. We are strongly confident with this result because initial RNA kinetic of early genes can only be explained by fast regulation at promoter level several minutes after stimulation. Proteins dynamics are way too slow to justify these early variations.

2. Twenty-two of the 29 inferred early genes are inhibited by the stimulus, while inhibitions are only present in 7 of the 28 non-early interactions. Thus inhibitions are overrepresented in stimulus-early genes interactions. An interpretation is that most of genes are auto-activated and their inhibition requires a strong and long enough signal to eliminate remaining auto-activated proteins. A constant and strong stimulus should be very efficient for this role like in [32] where stimulus long duration and high amplitude is required to overcome an auto-activation feedback effect. It could be very interesting in that respect to assess how the network would respond to a temporary stimulus, mimicking the commitment experiment described in [37] or [58].

3. None of our GRN candidates do contain so-called "hubs genes" affecting in parallel many genes, whereas existing GRN inferred generally present consequent hubs $[26,28,29,35]$. A possible interpretation is that hub identifications is mostly a by-product of correlation analysis. This interpretation is in line with the sparse nature of 
our candidate networks, as compared to some previous network (see e.g. [25] or [59]). This strongly departs with the assumption that small-world network might represent "universal laws" [60].

4. In order to reproduce non-monotonous gene expression variations, WASABI inferred systematically incoherent feedforward pattern instead of "simpler" negative feedback. This result is interesting because nothing in WASABI explain this bias since in silico benchmarking proved that WASABI is able to infer simple negative feedbacks (Fig. 4). Such "paradoxical components" have been proposed to provide robustness, generate temporal pulses, and provide fold-change detection [61].

5. WASABI candidates are limited in network depth by a maximum of 3 levels. We did not include readout genes during inference but addition of these genes would only increase GRN candidate depth by one level. GRN realistic candidates depth are thus limited by 4 levels. This might be due to the fact that information can only be relayed by limited number of intermediaries because of induced time delay, damping and noise. Indeed, general mechanism of molecules production/degradation behaves exactly as a low pass filter with a cutting frequency equivalent to the molecule degradation rate. Furthermore, protein information will be transmitted at the promoter target level by modulation of burst size and frequency, which are stochastic parameters, thereby adding noise to the original signal.

Such a strong limitation for information carrying capacity in GRN is at stake with long differentiation sequences, say from the hematopoietic stem cell to a fully committed cell. In such a case, tens of genes will have to be sequentially regulated. This might be resolved by the addition of auto-positive feedbacks. Such auto-positive feedbacks will create a dynamic memory whereby the information is maintained even in the absence of the initial information. An important implication is the loss of correlation between auto-activated gene and its regulator gene. Consequently, all algorithms based on stationary RNA single-cell correlation $[26,27]$ will hardly catch regulators of auto-activated genes.

Considering the importance of auto-positive feedback benefits on GRN information transfert, it is therefore not surprising to see that more than $80 \%$ of our GRN genes present auto-positive feedback signatures in their RNA distribution. Moreover, experimentally observed auto-positive feedback influence is stronger in our in vitro model than in our in silico models. Such a strong prevalence of auto-positive feedbacks has also been observed in a network underlying germ cell differentiation [59]. As mentioned earlier, care should be taken in interpreting such positive influences, which very likely rely on indirect influences, like epigenomic remodeling.

\section{Conclusions}

Inferring the structure of GRN is an inverse problem which has occupied the systems biology community for decades. This last few years, with the arrival of single cell transcriptomic data, many GRN inference algorithms based on the analysis of these data have been developed. Despite their contributions and successes, these approaches are confronted to some limitations such as:

- restriction to correlations which impairs predictive ability

- restriction to transcription factors to target gene interactions

- mono-data type, namely transcriptomic, ignoring protein level regulation

- biological over-simplifying assumptions induced by mathematical tools

Our work aims to provide a significant innovation in GRN inference problem to tackle these issues. We propose a divide-and-conquer strategy called WASABI, which splits the potentially untractable global problem into much simpler subproblems. We show that by adding one gene at a time, we can infer small networks, the behavior of which has been simulated in silico using a mechanistic model which incorporates the fundamentally probabilistic nature of the gene expression process. When applied to real-life data, our algorithm sheds a new fascinating light onto the molecular control of a differentiation process. GRN candidates were generated with a very interesting common topology which stands apart from typical literature and which is biologically relevant regarding several aspects as the very central role of the stimulus, the absence of "hub genes", the limitation in network depth and the presence of many auto-activation loops.

Together, these results demonstrate WASABI ability to tackle some general GRN inference issues:

- inference of causalities even in case of feedbacks

- definition of functional interactions underlying indirect regulations as post-translational regulation or nuclear translocation

- capability to integrate proteomic data to reproduce translational or post-translational regulation (observed in $50 \%$ of our genes)

- versatility and computational tractability using HPC facilities enabled by WASABI original iterative process

We believe that WASABI should be of great interest for biologists interested in GRN inference, and beyond for those aiming at a dynamical network view of their 
biological processes. We are convinced that this could really advance the field, opening an entire new way of analyzing single cell data for GRN inference.

\section{Methods}

\section{Mechanistic GRN model}

Our approach is based on a mechanistic model that has been previously introduced in [36] and which is summedup in Fig. 7.

In all that follows, we consider a set of $G$ interacting genes potentially influenced by a stimulus level $Q$. Each gene $i$ is described by its promoter state $E_{i}=0$ (off) or 1 (on), its mRNA level $M_{i}$ and its protein level $P_{i}$. We recall the model definition in the following equation, together with notations that will be extensively used throughout this article.

$$
\left\{\begin{aligned}
E_{i}(t) & : 0 \stackrel{k_{\text {on }}}{\rightarrow} 1, \quad 1 \stackrel{k_{\text {off }}}{\rightarrow} 0 \\
M_{i}^{\prime}(t) & =s_{0, i} E_{i}(t)-d_{0, i} M_{i}(t) \\
P_{i}^{\prime}(t) & =s_{1, i} M_{i}(t)-d_{1, i} P_{i}(t)
\end{aligned}\right.
$$

The first line in model (1) represents a discrete, Markov random process, while the two others are ordinary differential equations (ODEs) describing the evolution of mRNA and protein levels. Interactions between genes and stimulus are then characterized by the assumption that $k_{\text {on }}$ and $k_{\text {off }}$ are functions of $P=\left(P_{1}, \ldots, P_{G}\right)$ and $Q$. The form for $k_{\text {on }}$ is the following (for $k_{\text {off }}$, replace $\theta_{i, j}$ by $-\theta_{i, j}$ ): \%beginlinenomath

$$
k_{\mathrm{on}}(P, Q)=\frac{k_{\mathrm{on} \_\min , i}+k_{\mathrm{on} \_\max , i} \beta_{i} \Phi_{i}(P, Q)}{1+\beta_{i} \Phi_{i}(P, Q)}
$$

$$
\Phi_{i}(P, Q)=\frac{1+e^{\theta_{i, 0}} Q}{1+Q} \prod_{j=1}^{G} \frac{1+e^{\theta_{i, j}}\left(\frac{P_{j}}{H_{j}}\right)^{\gamma}}{1+\left(\frac{P_{j}}{H_{j}}\right)^{\gamma}}
$$

This interaction function slightly differs from [36] since auto-feedback is considered as any other interactions and stimulus effect is explicitly defined. Exponent parameter $\gamma$ is set to default value 2 . Interaction threshold $H_{j}$ is associated to protein $j$. Interaction parameters $\theta_{i, j}$ will be estimated during the iterative inference. Parameter $\beta_{i}$ corresponds to GRN external and constant influence on gene to define its basal expression: it is computed at simulation initialization in order to set $k_{\text {on }}$ and $k_{\text {off }}$ to their initial value. From now on, we drop the index $i$ to simplify our notation when there is no ambiguity.

\section{Overview of WASABI workflow}

WASABI framework is divided in 3 main steps as described in Fig. 8. First, individual gene parameters defined in model (1) (all except $\theta$ and $H$ ) are estimated before network inference from a number of experimental data types acquired during T2EC differentiation. They include time stamped single-cell transcriptomic [37], bulk transcription inhibition kinetic [37] and bulk proteomic data [39]. In a second step, genes are sorted regarding their wave times (see "Results" section for a description of wave concept) estimated from the mean of single cell transcriptomic data for promoter waves, and bulk proteomic data for protein waves. Finally, network iterative inference step is performed from single transcriptomic data, previously inferred gene parameters and sorted genes list. All methods are detailed in following sections, an overview of workflow is given by Fig. 8 .

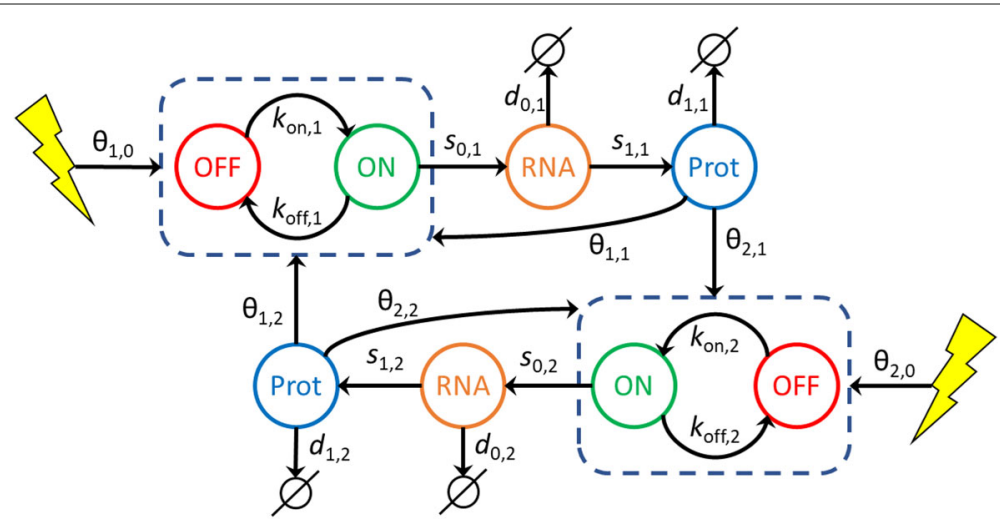

Fig. 7 GRN mechanistic and stochastic model. Our GRN model is composed of coupled piecewise deterministic Markov processes. In this example 2 genes are coupled. A gene $i$ is represented by its promoter state (dashed box) which can switch randomly from ON to OFF, and OFF to ON, respectively at $k_{\mathrm{on}, i}$ and $k_{\mathrm{off}, i}$ mean rate. When promoter state is $\mathrm{ON}, \mathrm{mRNA}$ molecules are continuously produced at a $s_{0, i}$ rate. mRNA molecules are constantly degraded at a $d_{0, i}$ rate. Proteins are constantly translated from mRNA at a $s_{1, i}$ rate and degraded at a $d_{1, i}$ rate. The interaction between a regulator gene $j$ and a target gene $i$ is defined by the dependence of $k_{\text {on }, i}$ and $k_{\text {off }, i}$ with respect to the protein level $P_{j}$ of gene $j$ and the interaction parameter $\theta_{i, j}$. Likewise, a stimulus (yellow flash) can regulate a gene $i$ by modulating its $k_{\text {on, } i}$ and $k_{\text {off }, i}$ switching rates with interaction parameter $\theta_{i, 0}$ 


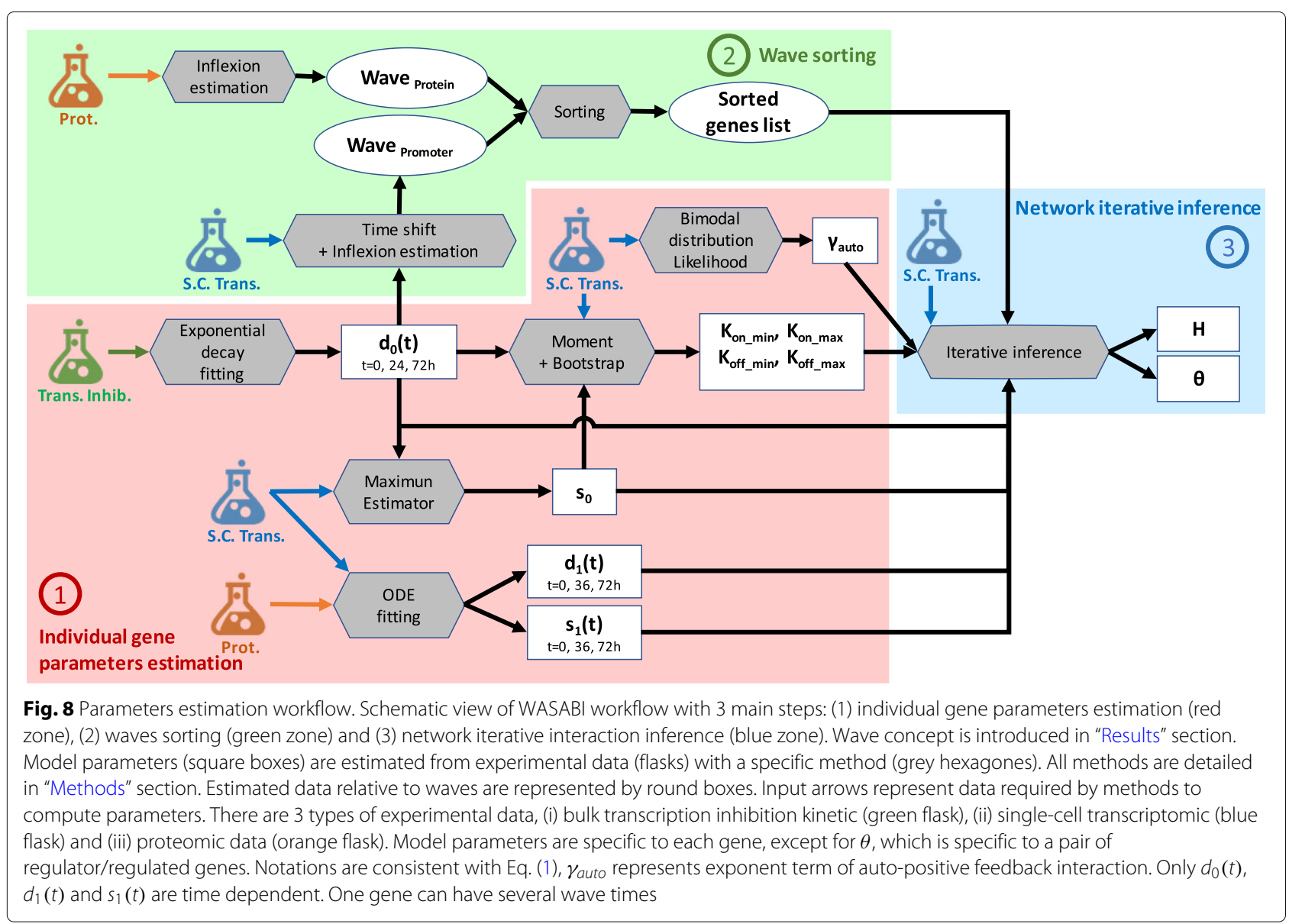

For T2EC in vitro application, tables of gene parameters and wave times are provided in supporting information. For in silico benchmarking we assume that gene parameters $d_{0}, d_{1}, s_{1}$ are known. Single-cell data and bulk proteomic data are simulated from in silico GRNs for time points $0,2,4,8,24,33,48,72$ and $100 \mathrm{~h}$.

\section{First step - Individual gene parameters estimation Exponential decay fitting for mRNA degradation rate $\left(d_{0}\right)$ estimation}

The degradation rate $d_{0}$ corresponds to active decay (i.e. destruction of mRNA) plus dilution due to cell division. The RNA decay was already estimated in [37] before differentiation (0h), $24 \mathrm{~h}$ and $72 \mathrm{~h}$ after differentiation induction from population-based data of mRNA decay kinetic using actinomycin D-treated T2EC (https://osf.io/k2q5b). Cell division dilution rate is assumed to be constant during the differentiation process and cell cycle time has been experimentally measured at $20 \mathrm{~h} \mathrm{[38].}$

\section{Maximum estimator for $m R N A$ transcription rate $\left(s_{0}\right)$ estimation}

To infer the transcription rate $s_{0}$, we used a maximum estimator based on single-cell expression data generated in [37]. We suppose that the highest possible mRNA level is given by $s_{0} / d_{0}$. Thus $s_{0}$ corresponds to the maximum mRNA count observed in all cells and time points multiplied by $\max _{t}\left(d_{0}(t)\right)$.

\section{Method of moments and bootstrapping for range of promoter switching rates $\left(\boldsymbol{k}_{\mathrm{on} / \mathrm{off} \min / \max }\right)$ estimation} Dynamic parameters $k_{\text {on }}$ and $k_{\text {off }}$ are bounded respectively by constant parameters $\left[k_{\text {on_min }} ; k_{\text {on_max }}\right]$ and [ $\left.k_{\text {off_min }} ; k_{\text {off_max }}\right]$ (see Eq. $\left.(2)\right)$ which are estimated as follows from time course single-cell transcriptomic data. Parameters $s_{0}$ and $d_{0}(t)$ are supposed to be previously estimated for each gene at time $t$.

Range parameters shall be compliant with constraints (Eq. (4)) imposed by the transcription dynamic regime observed in vitro. RNA distributions [37] have many zeros, which is consistent with the bursty regime of transcription. There is no observed RNA saturation in distributions. Moreover, all GRN parameters should also comply with computational constraints. On the one hand, the time step $d t$ used for simulations shall be small enough regarding GRN dynamics to avoid aliasing (undersampling) effects. On the other hand, $d t$ should not be too small to save computation time. These constraints correspond to 


$$
k_{\text {on }}<d_{0}<k_{\text {off }}<\frac{1}{d t}
$$

and we deduce inequalities for ranges:

$k_{\text {on_min }}<k_{\text {on_max }}<d_{0}<k_{\text {off_min }}<k_{\text {off_max }}<\frac{1}{d t}$.

We set the default value $k_{\text {on_min }}$ to $0.001 \mathrm{~h}^{-1}$. Parameter $k_{\text {on_max }}$ is estimated from time course single-cell transcriptomic data after removing zeros. This truncation mimics a distribution where gene is always activated, so that $k_{\text {on }}$ is close to its maximum value $k_{\text {on_max }}$. With these truncated distributions, for each time point $t$, we estimate $k_{\text {on }, t}$ using a moment-based method defined in [62]. We bootstrapped 1000 times to get a list of $k_{\text {on, } t, n}$ with index $n$ corresponding to bootstrap sample $n$. For each time point we compute the $95 \%$ percentile of $k_{\text {on, }, n}$, then we consider the mean value of these percentiles to have a first estimate of $k_{\text {on_max }}$. This $k_{\text {on } \max }$ is then down and up limited respectively between $k_{\text {on_max_lim_min }}$ and $k_{\text {on_max_lim_max }}$ given in Eq. (6) to guarantee that observed $k_{\text {on }}$ can be easily reached during simulations with reasonable values of protein level (because of asymptotic behavior of interaction function). In other words $k_{\text {on } \max }$ shall not be too close from minimum or maximum observed $k_{\text {on }}$ considering $10 \%$ margins. Finally, this limited $k_{\text {on_max }}$ is up-limited by $0.5 \times \max _{t}\left(d_{0}(t)\right)$ to guarantee a $50 \%$ margin with $d_{0}(t)$.

$\begin{aligned} k_{\text {on_max_lim_min }} & =\frac{\max _{t}\left(\operatorname{median}_{n}\left(k_{\mathrm{on}, t, n}\right)\right)-0.1 \times k_{\text {on_min }}}{0.9} \\ k_{\text {on_max_lim_max }} & =\frac{\max _{t}\left(\operatorname{median}\left(k_{\mathrm{on}, t, n}\right)\right)-0.9 \times k_{\text {on_min }}}{0.1}\end{aligned}$

Parameter $k_{\text {off_min }}$ is set to $\max \left(d_{0}(t)\right)$ to comply with equation Eq. (5). Parameter $k_{\text {off_max }}^{t}$ is estimated like $k_{\text {on_max }}$ from time course single-cell transcriptomic data but without zero truncation.For each time point $t$, we estimate $k_{\text {off, } t}$ using a moment-based method defined in [62]. We bootstrapped 1000 times to get a list of $k_{\text {off,t,n }}$ with index $n$ corresponding to bootstrap sample $n$. For each time point we compute the $95 \%$ percentile of $k_{\text {off, } t, n}$, then we consider the mean value of these percentiles to have a first estimate of $k_{\text {off_max }}$. This $k_{\text {off_max }}$ is then down and up limited respectively between $k_{\text {off_max_lim_min }}$ and $k_{\text {off_max } \lim \_ \text {max }}$ given in Eq. (7) to guarantee that observed $k_{\text {off }}$ can be easily reached during simulations with reasonable values of protein level (because of asymptotic behavior of interaction function). In other words $k_{\text {off_max }}$ shall not be too close from minimum or maximum observed $k_{\text {off }}$ considering $10 \%$ margins. Finally, this limited $k_{\text {off } \max }$ is up-limited by $1 / d t$ to guaranty simulation anti-aliasing. $k_{\text {off_max_lim_min }}=\frac{\max _{t}\left(\underset{n}{\operatorname{median}}\left(k_{\text {off }, t, n}\right)\right)-0.1 \times k_{\text {off_min }}}{0.9}$

$k_{\text {off_max_lim_max }}=\frac{\max _{t}\left(\underset{n}{\operatorname{median}}\left(k_{\text {off }, t, n}\right)\right)-0.9 \times k_{\text {off_min }}}{0.1}$

\section{ODE fitting for protein translation and degradation rates} $\left(d_{1}, s_{1}\right)$ estimation

Rates $d_{1}(t)$ and $s_{1}(t)$ are estimated from comparison of proteomic population kinetic data [39] with RNA mean value kinetic data computed from single-cell data [37]. Parameter $d_{1}(t)$ corresponds to protein active decay rate while total protein degradation rate $d_{1 \_t o t}(t)$ includes decay plus cell division dilution. Associated total protein half-life is referred to as $t_{1 \_t} t(t)$. Parameters $s_{1}(t)$ and $d_{1 \_t o t}(t)$ are estimated using a calibration algorithm based on a maximum likelihood estimator (MLE) from package [63]. Objective function is given by the Root Mean Squared Error function (provided by the package) comparing experimental protein counts with simulated ones given by ODEs from our model (1) with RNA level provided by experimental mean RNA data:

$$
P^{\prime}(t)=s_{1}(t) M(t)-d_{1}(t) P(t)
$$

Fifty two out of our 90 selected genes were detected in proteomic data. 23 of these fit correctly experimental data with a constant $d_{1}$ and $s_{1}$ during differentiation. 5 genes were estimated with a variable $s_{1}(t)$ and a constant $d_{1}$ to fit a constant protein level with a decreasing RNA level. For the remaining 24 genes, protein level decreased while RNA is constant, which is modeled with $s_{1}$ constant and $d_{1}(t)$ variable.

For the genes that were not detected in our proteomic data we turned to the literature [64] and found 13 homologous genes with associated estimation of $d_{1}$ and $s_{1}$. For the remaining 25 genes, we estimated parameters with the following rationale: we consider that the non-detection in the proteomic data is due to low protein copy number, lower than 100. Moreover [64] proposed an exponential relation between $s_{1}$ and the mean protein level that we confirmed with our data (see supporting information), resulting in the following definition:

$$
s_{1}=10^{-1.47} \times P^{0.81}
$$

Linear regression was performed using the Python scipy.stats.linregress() method from Scipy package with the following parameters: $r^{2}=0.55$, slope $=0.81$, intercept $=-1.47$ and $p=2.97 \times 10^{-9}$. Therefore, if we extrapolate this relation for low protein copy numbers assuming $P<100$ copies, $s_{1}$ should be lower than 1 molecule/RNA/hour. Assuming the relation 


$$
\text { Prot }=\text { RNA } \times \frac{s_{1}}{d_{1 \_t o t}}
$$

between mean protein and RNA levels, we deduced a minimum value of $d_{1}$ from mean RNA level given by: $d_{1}>$ RNA/100. We set $s_{1}$ and $d_{1}$ respectively to their maximum and minimum estimated values.

\section{Bimodal distribution likelihood for auto-positive feedback exponent ( $\gamma_{\text {auto }}$ ) estimation}

We inferred the presence of auto-positive feedback by fitting an individual model for each gene, based on [36]. The model is characterized by a Hill-type power coefficient. The value of this coefficient was inferred by maximizing the model likelihood, available in explicit form. The key idea is that genes with auto-positive feedback typically show, once viewed on an appropriate scale, a strongly bimodal distribution during their transitory regime. The interested reader may find some details in the Additional file 1 of [36], especially in sections 3.6 and 5.2. Note that such auto-positive feedback may reflect either a direct auto-activation, or a strong but indirect positive loop, potentially involving other genes. Estimated Hilltype power coefficients for in silico and in vitro networks are provided in supporting information.

\section{Second step - Waves sorting}

\section{Inflexion estimator for wave time estimation}

Wave time for gene promoter $W_{\text {prom }}$ and protein $W_{\text {prot }}$ are estimated regarding their respective mean trace $\bar{E}$ and $\bar{P}$. Estimation differs depending on mean trace monotony. In vitro wave times are provided in supporting information.

1) If the mean trace is monotonous (checked manually), it is smoothed by a 3rd order polynomial approximation using method poly1d() from python numpy package. Wave time is then defined as the inflection time point of polynomial function where $50 \%$ of evolution between minimum and maximum is reached.

2) If the mean trace is not monotonous, it is approximated by a piecewise-linear function with 3 breakpoints that minimizes the least square error. Linear interpolations are performed using the polynomial.polyfit() function from python numpy package. Selection of breakpoints is performed using optimize.brute() function from python numpy package.

We obtained a series of 4 segments with associated breakpoints coordinate and slope. Slopes are thresholded: if absolute value is lower than 0.2 it is considered null. Then, we looked for inflection break times where segments with non null slope have an opposite sign compare to the previous segment, or if previous segment has a null slope. Each inflection break time corresponds to an initial effect of a wave. A valid time, when wave effect applies, is associated and corresponds to next inflection break time or to the end of differentiation. Thus, we obtained couples of inflection break time and valid time which defined the temporal window of associated wave effect. For each wave window, if mean trace variation between inflection break time and valid time is large enough (i.e., greater than $20 \%$ of maximal variation during all differentiation process for the gene), a wave time is defined as the time where half of mean trace variation is reached during wave time window.

Protein mean trace $\bar{P}$ is given by proteomic data if available, else it is computed from simulation traces with 500 cells using the model with the parameters estimated earlier. Promoter mean trace $\bar{E}$ is computed as follows from mean RNA trace (from single-cell transcriptomic data) with time delay correction induced by mRNA degradation rate $d_{0}$.

$$
\begin{aligned}
& \bar{E}(t)=\frac{k_{\text {on }}(t)}{k_{\text {on }}(t)+k_{\text {off }}(t)} \\
& \bar{E}\left(t-\frac{1}{d_{0}(t)}\right)=\frac{d_{0}}{s_{0}} \times \bar{M}(t) \times\left(t-\frac{1}{d_{0}(t)}\right)
\end{aligned}
$$

\section{Genes sorting}

Genes are sorted regarding their promoter waves time $W_{\text {prom }}$. Genes with multiple waves, in case of feedback for example, are present several times in the list. Moreover, genes are classified by groups regarding their position in the network. Genes directly regulated by the stimulus are called the early genes; Genes that regulates other genes are defined as regulatory genes; Genes that do not influence other genes are identified as readout genes. Note that genes can belong to several group.

We can deduce the group type for each gene from its wave time estimation. Subsequent constraints have been defined from in silico benchmarking (see "Results" section). A gene $i$ belongs to one of these groups according to following rules:

- if $W_{\text {prom }}<5 \mathrm{~h}$ then it is an early gene

- if $W_{\text {prom }}<7 \mathrm{~h}$ then it could be an early gene or another types

- if $\max _{i}\left(W_{\text {prom }, i}\right)+30 \mathrm{~h}<W_{\text {prot }}$ then it is a readout gene

- else it could be a regulatory or a readout gene

\section{Third step - Network iterative inference Interaction threshold $(H)$}

Interaction threshold $H$ is estimated for each protein. It corresponds to mean protein level at $25 \%$ between minimum and maximum mean protein level observed during differentiation by in silico simulations:

$$
H=P_{\min }+0.25\left(P_{\max }-P_{\min }\right)
$$

We choose the value of $25 \%$ to maximize the amplitude variation of $k_{\text {on }}$ and $k_{\text {off }}$ of gene target induced by the shift of the regulator protein level from its minimal to maximal value (see Eq. (2)). 


\section{Iterative calibration algorithm $\left(\theta_{i, j}\right)$}

The following algorithm gives a global overview of the iterative inference process:

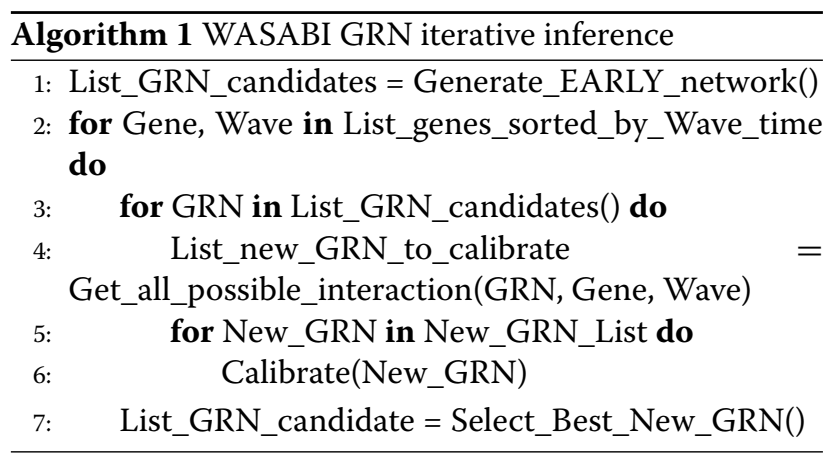

Generate_EARLY_network(): In a first step we calibrate the interactions between early genes and stimulus $\left(\theta_{i, 0}\right)$ to obtain an initial sub-GRN. Calibration algorithm Calibrate() is defined below.

List_genes_sorted_by_Wave_time: This list is computed prior to iterative inference (see previous subsection).

Get_all_possible_interaction(GRN, Gene, Wave): For each GRN candidate we estimate all possible interactions with the new gene and prior regulatory genes, or stimulus, regarding their respective promoter wave and protein wave with the following logic: if promoter wave is lower than $7 \mathrm{~h}$, interaction is possible between stimulus and the new gene. If the difference of promoter wave minus protein wave is between $-20 \mathrm{~h}$ and $+30 \mathrm{~h}$, then there is a possible interaction between the new gene and regulatory gene. Note: if WASABI is run in "directed" mode, only the true interaction is returned.

Calibrate(New_GRN): For interaction parameter calibration we used a Maximum Likelihood Estimator (MLE) from package spotpy [63]. The goal is to fit simulated single-cell gene marginal distribution with in vitro ones tuning efficiency interaction parameter $\theta_{i, j}$. For in silico study we defined GRN Fit distance as the mean of the 3 worst gene-wise fit distances. For in vitro study we defined GRN Fit distance as the mean of the fit distances of all genes. Gene-wise fit distance is defined as the mean of the 3 higher Kantorovitch distances[42] among time points. For a given time point and a given gene, the Kantorovitch fit distance corresponds to a distance between marginal distributions of simulated and experimental expression data. At the end of calibration the set of interaction parameter $\theta_{i, j}$ with associated GRN Fit distance is returned.
Select_Best_New_GRN() We fetch all GRN calibration fitting outputs from remote servers and select best new GRNs to be expanded for next iteration updating list of List_GRN_candidate. New networks candidates are limited by number of available computational cores.

\section{GRN simulation}

We use a basic Euler solver with fixed time step $(d t=$ $0.5 \mathrm{~h}$ ) to solve mRNA and protein ODEs [36]. The promoter state evolution between $t$ and $t+d t$ is given by a Bernoulli distributed random variable

$$
E(t+d t)=\operatorname{Bernoulli}(p(t))
$$

drawn with probability $p(t)$ depending on current $k_{\text {on }}, k_{\text {off }}$ and promoter state:

$$
p(t)=E(t) e^{-d t\left(k_{\mathrm{on}}+k_{\mathrm{off}}\right)}+\frac{k_{\mathrm{on}}}{k_{\mathrm{on}}+k_{\mathrm{off}}}\left(1-e^{-d t\left(k_{\mathrm{on}}+k_{\mathrm{off}}\right)}\right) .
$$

Time-dependent parameters like $d_{0}, d_{1}$ and $s_{1}$ are linearly interpolated between 2 points. The stimulus $Q$ is represented by a step function between 0 and 1000 at $t=0 \mathrm{~h}$. Simulation starts at $t=-60 \mathrm{~h}$ to ensure convergence to steady state before the stimulus is applied. Parameters $k_{\text {on }}$ and $k_{\text {off }}$ are given by Eq. (2).

\section{Additional file}

Additional file 1: Additional file with several tables and figures related to in vitro genes parameters and waves estimation, in silico benchmarking and in vitro GRN candidates fit distance distribution. (PDF $312 \mathrm{~kb}$ )

\section{Abbreviations}

DOE: Design of experiments; GRN: Gene regulatory network; HPC: High paralle computing; TF: Transcription factor; WASABI: WAveS analysis based inference

\section{Acknowledgements}

We thank the computational center of IN2P3 (Villeurbanne/France), specially Pascal Calvat, for access to HPC facilities; Eddy Caron (Avalon, ENS Lyon/INRIA) for his support on parallel computing implementation; Patrick Mayeux for proteomic data; and Rudiyanto Gunawan (ETH, Zürich) for critical reading of the manuscript. We would like to thank all members of the SBDM team,

Dracula team, and Camilo La Rota (Cosmotech) for enlightening discussions, We also thank the BioSyL Federation and the Ecofect Labex

(ANR-11-LABX-0048) of the University of Lyon for inspiring scientific events.

\section{Funding}

This work was supported by funding from the French agency ANR (ICEBERG; ANR-IABI-3096 and SinCity; ANR-17-CE12-0031) and the Association Nationale de la Recherche Technique (ANRT, CIFRE 2015/0436). The funders had no role in study design, data collection and analysis, decision to publish, or preparation of the manuscript.

\section{Availability of data and materials}

Single-cell transcriptomic data are available from [37]. Proteomic data are available from [39]. In silico generated data are available at https://osf.io/ gkedt/.

\section{Authors' contributions}

$A B, U H, P A G$ and $O G$ designed the study. AB performed the theoretical derivations, implemented the algorithms and conceived/analyzed the in silico study. UH implemented the algorithm for auto-positive feedback exponent estimation. AR, SG and AG participated in data generation. OG secured the 
funding. AB drafted the paper. UH, AR, AG, SG, PAG and OG revised the paper. All authors read and approved the final manuscript.

\section{Ethics approval and consent to participate}

Not applicable.

\section{Consent for publication}

Not applicable.

\section{Competing interests}

The results of this work will be exploited within the frame of a new company VIDIUM for which AB will serve as CSO.

\section{Publisher's Note}

Springer Nature remains neutral with regard to jurisdictional claims in published maps and institutional affiliations.

\section{Author details}

${ }^{1}$ University Lyon, ENS de Lyon, University Claude Bernard, CNRS UMR 5239, INSERM U1210, Laboratory of Biology and Modelling of the Cell, Lyon, France ${ }^{2}$ Inria Team Dracula, Inria Center Grenoble Rhône-Alpes, Lyon, France.

${ }^{3}$ Cosmotech, Lyon, France. ${ }^{4}$ Univ Lyon, Université Claude Bernard Lyon 1. CNRS UMR 5208, Institut Camille Jordan, Villeurbanne, France.

Received: 15 March 2019 Accepted: 9 April 2019

Published online: 02 May 2019

\section{References}

1. MacNeil LT, Walhout AJ. Gene regulatory networks and the role of robustness and stochasticity in the control of gene expression. Genome Res. 2011;21(5):645-57.

2. Greene JA, Loscalzo J. Putting the patient back together - social medicine, network medicine, and the limits of reductionism. N Engl J Med. 2017:377(25):2493-9. https://doi.org/10.1056/NEJMms1706744.

3. Sugimura R, Jha DK, Han A, Soria-Valles C, da Rocha EL, Lu Y-F, Goettel JA Serrao E, Rowe RG, Malleshaiah M, Wong I, Sousa P, Zhu TN, Ditadi A, Keller G, Engelman AN, Snapper SB, Doulatov S, Daley GQ. Haematopoietic stem and progenitor cells from human pluripotent stem cells. Nature. 545:432. https://doi.org/10.1038/nature22370.

4. Lis R, Karrasch CC, Poulos MG, Kunar B, Redmond D, Duran JGB, Badwe CR, Schachterle W, Ginsberg M, Xiang J, Tabrizi AR, Shido K, Rosenwaks Z, Elemento O, Speck NA, Butler JM, Scandura JM, Rafii S. Conversion of adult endothelium to immunocompetent haematopoietic stem cells. Nature. 545:439. https://doi.org/10.1038/nature22326.

5. leda M, Fu J-D, Delgado-Olguin P, Vedantham $V$, Hayashi $Y$, Bruneau BG, Srivastava D. Direct reprogramming of fibroblasts into functional cardiomyocytes by defined factors. Cell. 2010;142(3):375-86

6. Madhamshettiwar PB, Maetschke SR, Davis MJ, Reverter A, Ragan MA. Gene regulatory network inference: evaluation and application to ovarian cancer allows the prioritization of drug targets. Genome Med. 2012;4(5): 41. https://doi.org/10.1186/gm340

7. Creixell $P$, Schoof EM, Erler JT, Linding R. Navigating cancer network attractors for tumor-specific therapy. Nat Biotechnol. 2012;30(9):842.

8. Chai LE, Loh SK, Low ST, Mohamad MS, Deris S, Zakaria Z. A review on the computational approaches for gene regulatory network construction. Comput Biol Med. 48:55-65. https://doi.org/10.1016/j.compbiomed.2014. 02.011.

9. Dojer N, Gambin A, Mizera A, Wilczyński B, Tiuryn J. Applying dynamic bayesian networks to perturbed gene expression data. BMC Bioinformatics. 2006;7(1):249. https://doi.org/10.1186/1471-2105-7-249.

10. Vinh NX, Chetty M, Coppel R, Wangikar PP. Gene regulatory network modeling via global optimization of high order dynamic bayesian networks. BMC Bioinf. 2012;27:2765-6.

11. Akutsu T, Miyano S, Kuhara S. Identification of genetic networks from a small number of gene expression pattern under the boolean model. Pac Symp Biocomput. 1999;4:17-28.

12. Saadatpour A, Albert R. Boolean modeling of biological regulatory networks: A methodology tutorial. Methods. 2013;62(1):3-12. https://doi. org/10.1016/j.ymeth.2012.10.012.
13. Zhao W, Serpedin E, Dougherty ER. Inferring gene regulatory networks from time series data using the minimum description length principle. Bioinformatics. 2006;22(17):2129-35. https://doi.org/10.1093/ bioinformatics/btl364.

14. Polynikins A, Hogan SJ, Bernardo M. Comparing different ode modelling approaches forgene regulatory networks. J Theor Biol. 2009;261:511-30.

15. Bansal M, Belcastro V, Ambesi-Impiombato A, Di Bernardo D. How to infer gene networks from protein profiles. Mol Syst Biol. 2007;3:1-10.

16. Svensson V, Vento-Tormo R, Teichmann S. Exponential scaling of single-cell rnaseq in the last decade. Nat Protoc. 2018;13:599-604.

17. Fiers M, Minnoye L, Aibar S, Bravo Gonzalez-Blas C, Kalender Atak Z, Aerts S. Mapping gene regulatory networks from single-cell omics data. Brief Funct Genomics. 2018. https://doi.org/10.1093/bfgp/elx046.

18. Babtie A, Chan TE, Stumpf MPH. Learning regulatory models for cell development from single cell transcriptomic data. Curr Opin Syst Biol. 2017:5:72-81.

19. Yvert G. 'particle genetics': treating every cell as unique. Trends Genet 2014;30(2):49-56. https://doi.org/10.1016/j.tig.2013.11.002

20. Dueck H, Eberwine J, Kim J. Variation is function: Are single cell differences functionally important?: Testing the hypothesis that single cell variation is required for aggregate function. Bioessays. 2016;38(2):172-80. https://doi.org/10.1002/bies.201500124

21. Symmons O, Raj A. What's luck got to do with it: Single cells, multiple fates, and biological nondeterminism. Mol Cell. 2016;62(5):788-802. https://doi.org/10.1016/j.molcel.2016.05.023.

22. Cannoodt R, Saelens W, Saeys Y. Computational methods for trajectory inference from single-cell transcriptomics. Eur J Immunol. 2016;46(11): 2496-506. https://doi.org/10.1002/eji.201646347.

23. Chen H, Guo J, Mishra SK, Robson P, Niranjan M, Zheng J. Single-cell transcriptional analysis to uncover regulatory circuits driving cell fate decisions in early mouse development. Bioinformatics. 2015;31(7):1060-6. https://doi.org/10.1093/bioinformatics/btu777.

24. Lim CY, Wang H, Woodhouse S, Piterman N, Wernisch L, Fisher J, Gottgens B. Btr: training asynchronous boolean models using single-cell expression data. BMC Bioinformatics. 2016;17(1):355. https://doi.org/10. 1186/s12859-016-1235-y.

25. Moignard V, Woodhouse S, Haghverdi L, Lilly AJ, Tanaka Y, Wilkinson AC, Buettner F, Macaulay IC, Jawaid W, Diamanti E, Nishikawa S, Piterman N, Kouskoff V, Theis FJ, Fisher J, Gottgens B. Decoding the regulatory network of early blood development from single-cell gene expression measurements. Nat Biotechnol. 33(3):269-76. https://doi.org/10.1038/ nbt.3154.

26. Matsumoto H, Kiryu H. Scoup: a probabilistic model based on the ornstein-uhlenbeck process to analyze single-cell expression data during differentiation. BMC Bioinformatics. 2016;17(1):232. https://doi.org/10. 1186/s12859-016-1109-3.

27. Cordero P, Stuart JM. Tracing co-regulatory network dynamics in noisy, single-cell transcriptome trajectories: World scientific; 2016, pp. 576-87. https://doi.org/10.1142/9789813207813-0053.

28. Sanchez-Castillo M, Blanco D, Tienda-Luna IM, Carrion MC, Huang Y. A bayesian framework for the inference of gene regulatory networks from time and pseudo-time series data. Bioinformatics. 2017. https://doi.org/ 10.1093/bioinformatics/btx605.

29. Matsumoto $\mathrm{H}$, Kiryu H, Furusawa $\mathrm{C}$, Ko MSH, Ko SBH, Gouda N, Hayashi T, Nikaido I. Scode: an efficient regulatory network inference algorithm from single-cell rna-seq during differentiation. Bioinformatics. 2017;33(15): 2314-21. https://doi.org/10.1093/bioinformatics/btx194.

30. Ocone A, Haghverdi L, Mueller NS, Theis FJ. Reconstructing gene regulatory dynamics from high-dimensional single-cell snapshot data. Bioinformatics. 2015;31(12):89-96. https://doi.org/10.1093/ bioinformatics/btv257.

31. Huang S. Non-genetic heterogeneity of cells in development: more than just noise. Development. 2009;136(23):3853-62. https://doi.org/10.1242/ dev.035139.

32. Sokolik C, Liu Y, Bauer D, McPherson J, Broeker M, Heimberg G, Qi LS Sivak DA, Thomson M. Transcription factor competition allows embryonic stem cells to distinguish authentic signals from noise. Cell Syst. 2015;1(2):117-29. https://doi.org/10.1016/j.cels.2015.08.001.

33. Munsky B, Trinh B, Khammash M. Listening to the noise: random fluctuations reveal gene network parameters. Mol Syst Biol. 2009:5:318. https://doi.org/10.1038/msb.2009.75. 
34. Moris N, Pina C, Arias AM. Transition states and cell fate decisions in epigenetic landscapes. Nat Rev Genet. 2016;17(11):693-703. https://doi. org/10.1038/nrg.2016.98.

35. Papili Gao N, Ud-Dean MSM, Gandrillon O, Gunawan R. Sincerities: Inferring gene regulatory networks from time-stamped single cell transcriptional expression profiles. 2016. https://doi.org/10.1101/089110.

36. Herbach U, Bonnaffoux A, Espinasse T, Gandrillon O. Inferring gene regulatory networks from single-cell data: a mechanistic approach. BMC Syst Biol. 2017;11:105. https://doi.org/10.1186/s12918-017-0487-0.

37. Richard A, Boullu L, Herbach U, Bonnaffoux A, Morin V, Vallin E, Guillemin A, Papili Gao N, Gunawan R, Cosette J, Arnaud O, Kupiec JJ, Espinasse T, Gonin-Giraud S, Gandrillon O. Single-cell-based analysis highlights a surge in cell-to-cell molecular variability preceding irreversible commitment in a differentiation process. PLOS Biol. 2016;14(12):1002585. https://doi.org/10.1371/journal.pbio.1002585.

38. Gandrillon O, Schmidt U, Beug H, Samarut J. Tgf-beta cooperates with tgf-alpha to induce the self-renewal of normal erythrocytic progenitors: evidence for an autocrine mechanism. Embo J. 1999;18(10):2764-81.

39. Leduc M, Gautier E-F, Guillemin A, Broussard C, Salnot V, Lacombe C, Gandrillon O, Guillonneau F, Mayeux P. Deep proteomic analysis of chicken erythropoiesis. bioRxiv. 2018. https://doi.org/10.1101/289728. https://www.biorxiv.org/content/early/2018/03/27/289728.full.pdf.

40. Liu Z, Tjian R. Visualizing transcription factor dynamics in living cells. J Cell Biol. 2018. https://doi.org/10.1083/jcb.201710038.

41. Lambert SA, Jolma A, Campitelli LF, Das PK, Yin Y, Albu M, Chen X, Taipale J, Hughes TR, Weirauch MT. The human transcription factors. Cell. 2018;172:650-65.

42. Baba A, Komatsuzaki T. Construction of effective free energy landscape from single-molecule time series. Proc Natl Acad Sci U S A. 2007;104(49): 19297-302. https://doi.org/10.1073/pnas.0704167104.

43. Chai LE, Loh SK, Low ST, Mohamad MS, Deris S, Zakaria Z. A review on the computational approaches for gene regulatory network construction. Comput Biol Med. 2014;48:55-65. https://doi.org/10.1016/j.compbiomed. 2014.02.011.

44. Hecker M, Lambeck S, Toepfer S, Van Someren E, Guthke R. Gene regulatory network inference: data integration in dynamic models-a review. Biosystems. 2009;96(1):86-103.

45. Chen S, Mar JC. Evaluating methods of inferring gene regulatory networks highlights their lack of performance for single cell gene expression data. BMC Bioinformatics. 2018;19(1):232. https://doi.org/10. 1186/s12859-018-2217-z.

46. Stolovitzky G, Monroe D, Califano A. Dialogue on reverse engineering assessment and methods. Ann N Y Acad Sci. 2007;1115(1):1-22.

47. Schaffter T, Marbach D, Floreano D. Genenetweaver: in silico benchmark generation and performance profiling of network inference methods. Bioinformatics. 2011;27(16):2263-70.

48. Fisher J, Henzinger TA. Executable cell biology. Nat Biotechnol. 2007;25(11):1239-49. https://doi.org/10.1038/nbt1356.

49. Woodhouse S, Piterman N, Wintersteiger CM, Gottgens B, Fisher J. Scns: a graphical tool for reconstructing executable regulatory networks from single-cell genomic data. BMC Syst Biol. 2018;12(1):59. https://doi.org/10. 1186/s12918-018-0581-y.

50. Bonnaffoux A, Caron E, Croubois H, Gandrillon O. A cloud-aware autonomous workflow engine and its application to gene regulatory networks inference. Presented at CLOSER 2018-8th International conference on Cloud computing and Service Science. Funchal; 2018. p. 1-8.

51. Olsen JV, Mann M. Status of large-scale analysis of post-translational modifications by mass spectrometry. Mol Cell Proteomics. 2013;12(12): 3444-52. https://doi.org/10.1074/mcp.0113.034181.

52. Manning KS, Cooper TA. The roles of rna processing in translating genotype to phenotype. Nat Rev Mol Cell Biol. 2017;18(2):102-14. https:// doi.org/10.1038/nrm.2016.139.

53. Mandic A, Strebinger D, Regali C, Phillips NE, Suter DM. A novel method for quantitative measurements of gene expression in single living cells. Methods. 2017;120:65-75. https://doi.org/10.1016/j.ymeth.2017.04.008.

54. Lin YT, Hufton PG, Lee EJ, Potoyan DA. A stochastic and dynamical view of pluripotency in mouse embryonic stem cells. PLoS Comput Biol. 2018;14(2):1006000. https://doi.org/10.1371/journal.pcbi.1006000.

55. Zheng GXY, Terry JM, Belgrader $P$, Ryvkin $P$, Bent ZW, Wilson R, Ziraldo SB, Wheeler TD, McDermott GP, Zhu J, Gregory MT, Shuga J,
Montesclaros L, Underwood JG, Masquelier DA, Nishimura SY, SchnallLevin M, Wyatt PW, Hindson CM, Bharadwaj R, Wong A, Ness KD, Beppu LW, Deeg HJ, McFarland C, Loeb KR, Valente WJ, Ericson NG, Stevens EA, Radich JP, Mikkelsen TS, Hindson BJ, Bielas JH. Massively parallel digita transcriptional profiling of single cells. Nat Commun. 2017;8:14049.

56. Ud-Dean SM, Gunawan R. Optimal design of gene knockout experiments for gene regulatory network inference. Bioinformatics. 2016;32(6):875-83. https://doi.org/10.1093/bioinformatics/btv672

57. Kreutz C, Timmer J. Systems biology: experimental design. FEBS J. 2009;276(4):923-42. https://doi.org/10.1111/j.1742-4658.2008.06843.x.

58. Semrau S, Goldmann J, Soumillon M, Mikkelsen TS, Jaenisch R, van Oudenaarden A. Lineage commitment revealed by single-cell transcriptomics of differentiating embryonic stem cells. 2016. https://doi. org/10.1101/068288.

59. Jang S, Choubey S, Furchtgott L, Zou LN, Doyle A, Menon V, Loew EB, Krostag AR, Martinez RA, Madisen L, Levi BP, Ramanathan S. Dynamics of embryonic stem cell differentiation inferred from single-cell transcriptomics show a series of transitions through discrete cell states. Elife. 2017;6:. https://doi.org/10.7554/eLife.20487.

60. Barabasi AL, Oltvai ZN. Network biology: understanding the cell's functional organization. Nat Rev Genet. 2004;5(2):101-13.

61. Hart $Y$, Alon U. The utility of paradoxical components in biological circuits. Mol Cell. 2013;49(2):213-21. https://doi.org/10.1016/j.molcel.2013.01.004.

62. Peccoud J, Ycart B. Markovian modelling of gene product synthesis. Theor Popul Biol. 1995;48:222-34

63. Houska T, Kraft P, Chamorro-Chavez A, Breuer L. Spotting model parameters using a ready-made python package. PLoS ONE. 2015;10(12): 0145180. https://doi.org/10.1371/journal.pone.0145180.

64. Schwanhausser B, Busse D, Li N, Dittmar G, Schuchhardt J, Wolf J, Chen W, Selbach M. Corrigendum: Global quantification of mammalian gene expression control. Nature. 2013;495(7439):126-7.

Ready to submit your research? Choose BMC and benefit from

- fast, convenient online submission

- thorough peer review by experienced researchers in your field

- rapid publication on acceptance

- support for research data, including large and complex data types

- gold Open Access which fosters wider collaboration and increased citations

- maximum visibility for your research: over $100 \mathrm{M}$ website views per year

At BMC, research is always in progress.

Learn more biomedcentral.com/submissions 